\title{
MULTI-CRITERIA GROUP DECISION-MAKING USING AN EXTENDED EDAS METHOD WITH INTERVAL TYPE-2 FUZZY SETS
}

\author{
Mehdi Keshavarz Ghorabaee, Maghsoud Amiri, \\ Edmundas Kazimieras Zavadskas, Zenonas Turskis
}

\section{Introduction}

Multi-criteria decision-making (MCDM), which is a sub-discipline of operations research (OR), includes many methods and problems, which are related to many fields such as economy, engineering, military and management (Zavadskas et al., 2014; Lazauskas et al., 2015; Tošenovský, 2015). There are different classifications of MCDM problems and methods. We can classify them as multi-criteria evaluation problems and multi-criteria design problems (Chakraborty et al., 2015; Triantaphyllou, 2013). The first class is also known as the multiattribute decision-making and consists of a finite number of alternatives known in the beginning of the decision-making process, and each alternative is represented by its performance in multiple criteria (Mardani et al., 2016; Faraji Sabokbar et al., 2016). The second class is also known as the multi-objective decision-making, and an alternative (solution) can be found by solving a mathematical model (Yu et al., 2016; Mahdiraji et al., 2016). This study focuses on the first class of MCDM problems.

Because of the characteristics of the decision-making problems, the uncertainty of information is usually inevitable in the MCDM problems. Fuzzy set theory is an efficient tool for modeling the human knowledge and evaluations which are used in the decision-making process (Stanujkic et al., 2015). To handle the uncertainty of multi-criteria decision-making problems, the weights of criteria and the performance of alternatives are usually characterized by type-1 fuzzy sets that proposed by Zadeh (1965). Many researchers have studied the MCDM methods and problems in the type-1 fuzzy environment (Ecer, 2015; Karabasevic et al., 2016; Aliakbari Nouri et al., 2015; Li et al., 2015; Razavi Hajiagha et al., 2015). Mardani et al. (2015) reviewed the MCDM methods and their applications in fuzzy environment.
Type-1 fuzzy sets are efficient in modeling the multi-criteria decision-making problems and have many applications for extending MCDM methods in an uncertain environment. However, we may confront with situations that more degrees of flexibility are needed to deal with the decision-making process. Type-2 fuzzy sets (T2FSs), which was introduced by Zadeh (1975), are more flexible than type-1 fuzzy sets in the modeling of uncertainty. Interval type-2 fuzzy sets (IT2FSs) are a special type of T2FSs. Some basic definitions of IT2FSs were proposed by Mendel et al. (2006). IT2FSs have increasingly been considered by researchers in applications and extensions of multi-criteria decision-making methods. Chen and Lee (2010) developed a new ranking method for interval type-2 fuzzy sets and used it in a new fuzzy MCDM method. Chen et al. (2012) proposed a new ranking method and a new multi-criteria decision-making method with interval type-2 fuzzy sets. Wang et al. (2012) introduced a multi-criteria group decision-making (MCGDM) method in type2 fuzzy environment, which can be used with incomplete information about criteria weights. $\mathrm{Hu}$ et al. (2013) developed a new ranking method based on the possibility degree for IT2FSs and applied it in multi-criteria decisionmaking process. Keshavarz Ghorabaee et al. (2014) presented a new fuzzy ranking method and extended COPRAS (Complex Proportional Assessment) method in the context of IT2FSs to evaluate suppliers in a supply chain. Celik et al. (2014) proposed an interval type-2 fuzzy MCDM method to identify and evaluate critical success factors for humanitarian relief logistics management. Balin and Baraçli (2015) developed a fuzzy MCDM methodology based on the IT2FSs for evaluating renewable energy alternatives in Turkey. Chen (2015) proposed a new likelihood-based interval type-2 fuzzy 
MCDM method using the concepts of likelihoodbased performance indices, likelihood-based comprehensive evaluation values, and signed distance-based evaluation values. Sang and Liu (2016) presented ranking method for IT2FSs and extended an IT2FSs-based TODIM method for green supplier selection in automobile manufacturers. Keshavarz Ghorabaee et al. (2016b) extended the WASPAS method with IT2FSs and applied it to evaluation of green suppliers. Celik et al. (2015) performed a comprehensive review of MCDM methods with interval type-2 fuzzy sets.

Keshavarz Ghorabaee et al. (2015b) introduced the evaluation based on distance from average solution (EDAS) method. This method was also extended for decision-making in fuzzy environment and was applied to supplier selection problem (Keshavarz Ghorabaee et al., 2016c). However, the previous versions of this method are not appropriate to deal with MCGDM problems with IT2FSs. In this study, we propose a new extended EDAS with interval type-2 fuzzy sets (EDAS-IT2FSs). A numerical example is employed to illustrate the process and show the effectiveness of the proposed method. A comparison and a sensitivity analysis are also performed to represent the validity and stability of the ranking result. The results of these analyses show that the proposed extended EDAS method is stable in different weights of criteria and well-consistent with some existing methods.
The rest of this paper is organized as follows. In Section 1, some basic concepts and arithmetic operation of T2FS are summarized. In Section 2, EDAS-IT2FSs is presented to deal with MCGDM with interval type-2 fuzzy sets. In Section 3, we use a numerical example to illustrate the procedure of using the EDASIT2FSs method. In Section 4, a sensitivity analysis is performed to show the validity and stability of the results of the proposed method. Finally, conclusions are discussed.

\section{Concepts and Arithmetic Operations}

Type-2 fuzzy sets are one of the main extensions of the type-1 fuzzy sets. T2FSs are represented by primary and secondary membership values. These types of fuzzy sets could be very useful in many fields of sciences, especially decisionmaking theory. In this section, the basic concepts and arithmetic operations of this type of fuzzy sets are defined.

Definition 1. A T2FS $\tilde{A}$ is described by a type-2 membership function, expressed as follows (Mendel et al., 2006):

$$
\tilde{A}=\int_{x \in X} \int_{u \in J_{x}} \mu_{\tilde{A}}(x, u) /(x, u)
$$

where $X$ denotes the domain of $\tilde{A}, \mu_{\tilde{A}}$ refers to the membership function (secondary membership function) of $\tilde{A}, J_{x} \subseteq[0,1]$ denotes the primary membership function and $\iint$ denotes the union over all admissible $x$ and $u$. For a T2FS $\tilde{A}$, if all $\mu_{\tilde{A}}(x, u)=1$, then $\tilde{A}$ is called interval type-2 fuzzy set.

\section{Fig. 1: An example of a trapezoidal IT2FS}

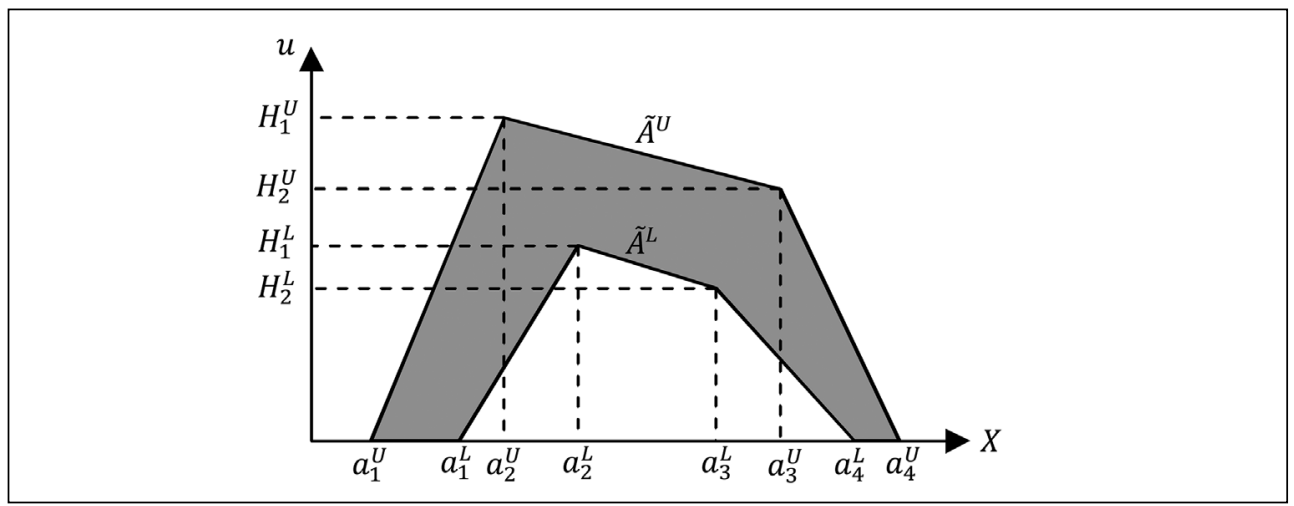

Source: Chen and Lee (2010) 


\section{Ekonomika a management}

Definition 2. An IT2FS is called trapezoidal IT2FS if and only if the UMF (Upper Membership Function) and the LMF (Lower Membership Function) are both trapezoidal fuzzy sets. Let $\tilde{A}$ be a trapezoidal IT2FS. $\tilde{A}$ can be expressed as follows (Chen and Lee, 2010):

$$
\begin{aligned}
& \tilde{A}=\left(\tilde{A}^{T} \mid T \epsilon\{U, L\}\right)=\left(a_{i}^{T} ; H^{T}{ }_{1 A} ; H_{2 A}^{T} \mid\right. \\
& \mid T \epsilon\{U, L\}, i=1,2,3,4)
\end{aligned}
$$

where $\tilde{A}^{U}$ and $\tilde{A}^{L}$ denote the UMF and LMF of $\tilde{A}$, respectively, and $H^{U}{ }_{j} \epsilon[0,1]$ and $H^{L}{ }_{j} \epsilon$ $[0,1](j=1,2)$ denote the membership values of the corresponding elements $a_{j+1}^{U}$ and $a^{L}$, respectively. An example of a trapezoidal IT2FS is shown in Fig. 1.

Definition 3. Suppose that $\tilde{\tilde{A}}$ and $\tilde{\tilde{B}}$ are two trapezoidal IT2FSs and $d$ is a crisp number where,

$$
\begin{aligned}
& \tilde{\tilde{A}}=\left(\tilde{A}^{T} \mid T \in\{U, L\}\right)=\left(a_{i}^{T} ; H_{1 A}^{T}, H_{2 A}^{T} \mid T \in\{U, L\}, i=1,2,3,4\right) \\
& \tilde{B}=\left(\tilde{B}^{T} \mid T \in\{U, L\}\right)=\left(b_{i}^{T} ; H_{1 B}^{T}, H_{2 B}^{T} \mid T \in\{U, L\}, i=1,2,3,4\right)
\end{aligned}
$$

Then the arithmetic operations of IT2FSs are defined as follows (Keshavarz Ghorabaee et al., 2015a; Keshavarz Ghorabaee et al., 2016a):

- Addition:

$$
\begin{aligned}
& \tilde{\tilde{A}} \oplus \tilde{\tilde{B}}=\left(a_{i}^{T}+b_{i}^{T} ; \min \left(H_{1 A}^{T}, H_{1 B}^{T}\right),\right. \\
& \left.\left.\min \left(H_{2 A}^{T}, H_{2 B}^{T}\right)\right) \mid T \in\{U, L\}, i=1,2,3,4\right) \\
& \tilde{A}+d=\left(a_{i}^{T}+d ; H_{1 A}^{T}, H_{2 A}^{T} \mid T \in\{U, L\}, i=1,2,3,4\right)
\end{aligned}
$$

- Subtraction:

$$
\begin{aligned}
& \tilde{\tilde{A}} \ominus \tilde{\tilde{B}}=\left(a_{i}^{T}-b_{5-i}^{T} ; \min \left(H_{1 A}^{T}, H_{1 B}^{T}\right),\right. \\
& \left.\min \left(H_{2 A}^{T}, H_{2 B}^{T}\right) \mid T \in\{U, L\}, i=1,2,3,4\right)
\end{aligned}
$$

- Multiplication:

$$
\begin{aligned}
& \tilde{\tilde{A}} \otimes \tilde{\tilde{B}}=\left(X_{i}^{T} ; \min \left(H_{1 A}^{T}, H_{1 B}^{T}\right),\right. \\
& \left.\min \left(H_{2 A}^{T}, H_{2 B}^{T}\right) \mid T \in\{U, L\}, i=1,2,3,4\right) \\
& X_{i}^{T}=\left\{\begin{array}{l}
\min \left(a_{i}^{T} b_{i}^{T}, a_{i}^{T} b_{5-i}^{T}, a_{5-i}^{T} b_{l}^{T}, a_{5-i}^{T} b_{5-i}^{T}\right) \text { if } i=1,2 \\
\max \left(a_{i}^{T} b_{i}^{T}, a_{i}^{T} b_{5-i}^{T}, a_{5-i}^{T} b_{i}^{T}, a_{5-i}^{T} b_{5-i}^{T}\right) \text { if } i=3,4
\end{array}\right.
\end{aligned}
$$

$$
\text { d. } \tilde{\tilde{A}}=\left\{\begin{array}{l}
\left(d . a_{i}^{T} ; H_{1 A}^{T}, H_{2 A}^{T} \mid T \in\{U, L\}, i=1,2,3,4\right) \text { if } d \geq 0 \\
\left(d . a_{5-i}^{T} ; H_{1 A}^{T}, H_{2 A}^{T} \mid T \in\{U, L\}, i=1,2,3,4\right) \text { if } d \leq 0
\end{array}\right.
$$

Definition 4. The crisp score of a trapezoidal IT2FS is defined as follows (Keshavarz Ghorabaee et al., 2015a):

$$
\mathbb{S}(\tilde{\tilde{A}})=\frac{1}{2}\left(\sum_{T \in\{u, L\}} \frac{a_{1}^{T}+\left(1+H_{1 A}^{T}\right) a_{2}^{T}+\left(1+H_{2 A}^{T}\right) a_{3}^{T}+a_{4}^{T}}{4+H_{1 A}^{T}+H_{2 A}^{T}}\right)
$$

Definition 5. A function is defined in the following to find the maximum between a trapezoidal interval type-2 fuzzy set fuzzy number and zero.

$$
Z(\tilde{\tilde{A}})=\left\{\begin{array}{lll}
\tilde{\tilde{A}} & \text { if } & \subseteq \\
\tilde{\tilde{0}} & \text { if } & \mathfrak{S}(\tilde{\tilde{A}})>0 \\
\tilde{A} & \leq 0
\end{array}\right.
$$

where $\tilde{\tilde{0}}=((0,0,0,0 ; 1,1),(0,0,0,0 ; 1,1))$.

\section{EDAS-IT2FSs Method}

The EDAS method was developed by Keshavarz Ghorabaee et al. (2015b). By comparing this method with some existing MCDM method, it was demonstrated that the EDAS method is efficient to handle decision-making problems with multiple criteria. In this section, an extended version of the EDAS method is proposed to deal with MCGDM problems in the interval type-2 fuzzy environment. The concepts and arithmetic operations of the IT2FSs, which has been presented in Section 1, are utilized for extending the EDAS method. The current study is focused on a situation that all evaluations of decision-makers are subjective. However, the proposed method can be used in a situation with both subjective and objective evaluations. Suppose that we have a set of $n$ alternatives $\left(\mathcal{A}=\left\{\mathcal{A}_{1}, \mathcal{A}_{2}, \ldots, \mathcal{A}_{n}\right\}\right)$, a set of $m$ criteria $\left(\mathcal{C}=\left\{\mathcal{C}_{1}, \mathcal{C}_{2}, \ldots, \mathcal{C}_{m}\right\}\right)$ and $k$ decision-makers $\left(\mathcal{D}=\left\{\mathcal{D}_{1}, \mathcal{D}_{2}, \ldots, \mathcal{D}_{k}\right\}\right)$. The steps of EDASIT2FSs method are presented as follows:

Step 1. Construct the average decision matrix $(X)$, shown as follows:

$$
X=\left[\tilde{\tilde{x}}_{i j}\right]_{n \times m}
$$

where,

$$
\tilde{\tilde{x}}_{i j}=\frac{1}{k} \underset{p=1}{\oplus} \tilde{\tilde{x}}_{i j}^{p}
$$

and $\tilde{\tilde{x}}_{i j}^{p}$ denotes the performance value of alternative $\mathcal{A}_{i}(1 \leq i \leq n)$ with respect to criterion $\mathcal{C}_{j}(1 \leq j \leq m)$ assigned by the $p$ th decision-maker $(1 \leq p \leq k)$.

Step 2. Construct the matrix of criteria weights, shown as follows: 


$$
W=\left[\widetilde{\widetilde{w}}_{j}\right]_{1 \times m}
$$

where,

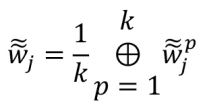

and $\widetilde{\widetilde{w}}_{j}^{p}$ denotes the weight of criterion $\mathcal{C}_{j}(1 \leq j \leq m)$ assigned by the $p$ th decisionmaker $(1 \leq p \leq k)$.

Step 3. Determine the matrix of average solutions, shown as follows:

$$
A V=\left[\widetilde{\widetilde{\mathcal{M}}}_{j}\right]_{1 \times m}
$$

where,

$$
\widetilde{\widetilde{M}}_{j}=\frac{1}{n} \underset{i=1}{\oplus} \tilde{\tilde{x}}_{i j}
$$

The elements of this matrix $\left(\widetilde{\widetilde{M}}_{j}\right)$ represents the average solutions with respect to each criterion. Therefore, the dimension of the matrix is equal to the dimension of criteria weights matrix.

Step 4. Suppose that $B$ is the set of beneficial criteria and $N$ is the set of non-beneficial criteria. In this step the matrices of positive distance from average $(P D A)$ and negative distance from average $(N D A)$ are calculated according to the type of criteria (beneficial and non-beneficial), shown as follows:

$$
\begin{aligned}
& P D A=\left[\tilde{\tilde{p}}_{i j}\right]_{n \times m} \\
& N D A=\left[\tilde{\tilde{n}}_{i j}\right]_{n \times m} \\
& \tilde{\tilde{p}}_{i j}=\left\{\begin{array}{l}
\frac{z\left(\tilde{\tilde{x}}_{i j} \ominus \widetilde{\widetilde{\mathcal{M}}}_{j}\right)}{\subseteq_{\left(\tilde{\mathcal{M}}_{j}\right)}} \quad \text { if } \quad j \in B \\
\frac{z\left(\widetilde{\widetilde{\mathcal{M}}}_{j} \ominus \tilde{\tilde{x}}_{i j}\right)}{\widetilde{S}_{\left(\tilde{\mathcal{M}}_{j}\right)}} \quad \text { if } \quad j \in N
\end{array}\right. \\
& \tilde{\tilde{n}}_{i j}=\left\{\begin{array}{l}
\frac{z\left(\tilde{\widetilde{\mathcal{M}}}_{j} \ominus \tilde{\tilde{x}}_{i j}\right)}{S_{\left(\tilde{\mathcal{M}}_{j}\right)}} \quad \text { if } \quad j \in B \\
\frac{z\left(\tilde{\tilde{x}}_{i j} \ominus \widetilde{\widetilde{M}}_{j}\right)}{\subseteq\left(\tilde{\widetilde{\mathcal{M}}}_{j}\right)} \quad \text { if } \quad j \in N
\end{array}\right.
\end{aligned}
$$

where $\tilde{\tilde{p}}_{i j}$ and $\tilde{\tilde{n}}_{i j}$ denote the positive and negative distance of performance value of $i$ th alternative from the average solution in terms of $j$ th criterion, respectively.

Step 5. Calculate the weighted sum of positive and negative distances for all alternatives, shown as follows:

$$
\begin{aligned}
& \widetilde{s p}_{i}= \underset{j=1}{m}\left(\widetilde{\widetilde{w}}_{j} \otimes \tilde{\tilde{p}}_{i j}\right) \\
& \widetilde{s n}_{i}=\underset{j=1}{m}\left(\widetilde{\widetilde{w}}_{j} \otimes \widetilde{\tilde{n}}_{i j}\right)
\end{aligned}
$$

Step 6. The normalized values of $\widetilde{\widetilde{s p}}_{i}$ and $\widetilde{\delta n}_{i}$ for all alternatives are calculated as follows:

$$
\begin{aligned}
& \widetilde{\widetilde{n p}}_{i}=\frac{\widetilde{\widetilde{s p}}_{i}}{\max _{i}\left(\subseteq\left(\widetilde{\widetilde{s p}}_{i}\right)\right)} \\
& \widetilde{\overline{n n}}_{i}=1-\frac{\widetilde{\widetilde{s n}}_{i}}{\max _{i}\left(\subseteq\left(\widetilde{\widetilde{s n}}_{i}\right)\right)}
\end{aligned}
$$

Step 7. Calculate the appraisal score $\left(\tilde{\tilde{\tilde{h}}}_{i}\right)$ for all alternatives, shown as follows:

$$
\tilde{\tilde{h}}_{i}=\frac{1}{2}\left(\widetilde{\widetilde{n p}}_{i} \oplus \widetilde{\widetilde{n n}}_{i}\right)
$$

Step 8. Rank the alternatives according to the decreasing ranking values of appraisal scores $(\mathcal{R V})$. In other words, the alternative with the highest appraisal score is the best choice among the candidate alternatives. It should be noted that the method proposed by Keshavarz Ghorabaee et al. (2014) is used in this step for computing the ranking value of trapezoidal IT2FSs.

\section{Numerical Example}

In this section, the application of the EDASIT2FSs method in an MCGDM problem is represented. For this aim, we use an example of multi-criteria evaluation of subcontractors in the construction industry. Suppose that a company, which is the main contractor involved in a construction project, intends to subcontract some parts of the project to a qualified subcontractor. The board of directors of the company performed an initial screening, and eight alternatives $\left(\mathcal{A}_{1}\right.$ to $\left.\mathcal{A}_{8}\right)$ remained for further appraisal. A group of three decisionmakers $\left(\mathcal{D}_{1}, \mathcal{D}_{2}\right.$ and $\left.\mathcal{D}_{3}\right)$ from the experts of the 


\begin{tabular}{c|c} 
Linguistic terms & Interval type-2 fuzzy sets \\
\hline Very low $(\mathrm{VL})$ & $((0,0,0,0.1 ; 1,1),(0,0,0,0.05 ; 0.9,0.9))$ \\
\hline Low $(\mathrm{L})$ & $((0,0.1,0.15,0.3 ; 1,1),(0.05,0.1,0.15,0.2 ; 0.9,0.9))$ \\
\hline Medium low $(\mathrm{ML})$ & $((0.1,0.3,0.35,0.5 ; 1,1),(0.2,0.3,0.35,0.4 ; 0.9,0.9))$ \\
\hline Medium $(\mathrm{M})$ & $((0.3,0.5,0.55,0.7 ; 1,1),(0.4,0.5,0.55,0.6 ; 0.9,0.9))$ \\
\hline Medium high $(\mathrm{MH})$ & $((0.5,0.7,0.75,0.9 ; 1,1),(0.6,0.7,0.75,0.8 ; 0.9,0.9))$ \\
\hline High $(\mathrm{H})$ & $((0.7,0.85,0.9,1 ; 1,1),(0.8,0.85,0.9,0.95 ; 0.9,0.9))$ \\
\hline Very high $(\mathrm{VH})$ & $((0.9,1,1,1 ; 1,1),(0.95,1,1,1 ; 0.9,0.9))$ \\
\hline
\end{tabular}

Source: Keshavarz Ghorabaee et al. (2014)

\section{Tab. 2: Performance values of alternatives}

\begin{tabular}{|c|c|c|c|c|c|c|c|c|}
\hline \multirow{2}{*}{ DMs } & \multirow{2}{*}{ Alternatives } & \multicolumn{7}{|c|}{ Criteria } \\
\hline & & $\mathcal{C}_{1}$ & $\mathcal{C}_{2}$ & $\mathcal{C}_{3}$ & $\mathcal{C}_{4}$ & $\mathcal{C}_{5}$ & $\mathcal{C}_{6}$ & $\mathcal{C}_{7}$ \\
\hline \multirow{8}{*}{$\mathcal{D}_{1}$} & $\mathcal{A}_{1}$ & $M$ & $\mathrm{ML}$ & $M$ & $\mathrm{M}$ & $\mathrm{MH}$ & $\mathrm{ML}$ & $\mathrm{VH}$ \\
\hline & $\mathcal{A}_{2}$ & $\mathrm{~L}$ & $\mathrm{MH}$ & $\mathrm{MH}$ & $\mathrm{VH}$ & $\mathrm{VH}$ & $\mathrm{H}$ & $\mathrm{M}$ \\
\hline & $\mathcal{A}_{3}$ & $\mathrm{VL}$ & $\mathrm{VH}$ & $\mathrm{MH}$ & $\mathrm{MH}$ & $\mathrm{H}$ & $\mathrm{H}$ & $\mathrm{M}$ \\
\hline & $\mathcal{A}_{4}$ & $\mathrm{H}$ & $\mathrm{VH}$ & $\mathrm{VH}$ & L & $\mathrm{H}$ & $\mathrm{MH}$ & $\mathrm{VL}$ \\
\hline & $\mathcal{A}_{5}$ & $\mathrm{VH}$ & $\mathrm{M}$ & $\mathrm{VH}$ & $\mathrm{L}$ & $\mathrm{MH}$ & $\mathrm{M}$ & $\mathrm{ML}$ \\
\hline & $\mathcal{A}_{6}$ & $\mathrm{VH}$ & $\mathrm{H}$ & $\mathrm{MH}$ & $\mathrm{L}$ & $\mathrm{VH}$ & $\mathrm{H}$ & $\mathrm{VL}$ \\
\hline & $\mathcal{A}_{7}$ & $\mathrm{M}$ & $\mathrm{MH}$ & $\mathrm{VH}$ & $\mathrm{H}$ & $\mathrm{ML}$ & $\mathrm{MH}$ & $\mathrm{MH}$ \\
\hline & $\mathcal{A}_{8}$ & $\mathrm{MH}$ & $\mathrm{L}$ & $\mathrm{ML}$ & $\mathrm{MH}$ & $\mathrm{VL}$ & M & $\mathrm{VH}$ \\
\hline \multirow{8}{*}{$\mathcal{D}_{2}$} & $\mathcal{A}_{1}$ & M & M & $\mathrm{ML}$ & M & $\mathrm{MH}$ & $\mathrm{L}$ & $\mathrm{H}$ \\
\hline & $\mathcal{A}_{2}$ & $\mathrm{ML}$ & $\mathrm{H}$ & $\mathrm{MH}$ & $\mathrm{H}$ & $\mathrm{H}$ & $\mathrm{MH}$ & $\mathrm{ML}$ \\
\hline & $\mathcal{A}_{3}$ & $\mathrm{~L}$ & $\mathrm{H}$ & $\mathrm{M}$ & $\mathrm{MH}$ & $\mathrm{MH}$ & $\mathrm{MH}$ & M \\
\hline & $\mathcal{A}_{4}$ & $\mathrm{H}$ & $\mathrm{VH}$ & $\mathrm{H}$ & L & $\mathrm{H}$ & M & $\mathrm{L}$ \\
\hline & $\mathcal{A}_{5}$ & $\mathrm{H}$ & $\mathrm{MH}$ & $\mathrm{VH}$ & $\mathrm{VL}$ & $\mathrm{M}$ & $M$ & $\mathrm{~L}$ \\
\hline & $\mathcal{A}_{6}$ & $\mathrm{VH}$ & $\mathrm{H}$ & $\mathrm{H}$ & $\mathrm{ML}$ & $\mathrm{VH}$ & $\mathrm{VH}$ & $\mathrm{VL}$ \\
\hline & $\mathcal{A}_{7}$ & $\mathrm{MH}$ & $\mathrm{MH}$ & $\mathrm{H}$ & $\mathrm{MH}$ & M & $\mathrm{M}$ & $\mathrm{M}$ \\
\hline & $\mathcal{A}_{8}$ & $\mathrm{H}$ & $\mathrm{ML}$ & $\mathrm{L}$ & $\mathrm{MH}$ & $\mathrm{ML}$ & $\mathrm{ML}$ & $\mathrm{MH}$ \\
\hline \multirow{8}{*}{$\mathcal{D}_{3}$} & $\mathcal{A}_{1}$ & $\mathrm{MH}$ & $\mathrm{ML}$ & $\mathrm{ML}$ & $\mathrm{ML}$ & $\mathrm{H}$ & $\mathrm{M}$ & $\mathrm{H}$ \\
\hline & $\mathcal{A}_{2}$ & $\mathrm{ML}$ & $\mathrm{MH}$ & $\mathrm{M}$ & $\mathrm{MH}$ & $\mathrm{VH}$ & $\mathrm{H}$ & $\mathrm{MH}$ \\
\hline & $\mathcal{A}_{3}$ & $\mathrm{ML}$ & $\mathrm{MH}$ & $M$ & $M$ & $\mathrm{MH}$ & $\mathrm{MH}$ & $M$ \\
\hline & $\mathcal{A}_{4}$ & $\mathrm{MH}$ & $\mathrm{H}$ & $\mathrm{H}$ & $L$ & $\mathrm{H}$ & $\mathrm{H}$ & $\mathrm{ML}$ \\
\hline & $\mathcal{A}_{5}$ & $\mathrm{H}$ & $\mathrm{MH}$ & $\mathrm{H}$ & $\mathrm{VL}$ & $\mathrm{H}$ & $\mathrm{MH}$ & $\mathrm{M}$ \\
\hline & $\mathcal{A}_{6}$ & $\mathrm{VH}$ & $\mathrm{H}$ & $\mathrm{H}$ & $\mathrm{VL}$ & $\mathrm{VH}$ & $\mathrm{VH}$ & $\mathrm{VL}$ \\
\hline & $\mathcal{A}_{7}$ & $M$ & $M$ & $\mathrm{MH}$ & $M$ & $M$ & $\mathrm{H}$ & $\mathrm{MH}$ \\
\hline & $\mathcal{A}_{8}$ & $M$ & $\mathrm{VL}$ & $L$ & $M$ & $L$ & $\mathrm{MH}$ & $\mathrm{VH}$ \\
\hline
\end{tabular}


company was formed for making a decision on these alternatives. These experts chose seven criteria $\left(\mathcal{C}_{1}\right.$ to $\left.\mathcal{C}_{7}\right)$ to appraise alternatives. The list of the selected criteria is represented as follows (Vahdani et al., 2013; Plebankiewicz, 2012; Abbasianjahromi et al., 2013):

- Management capability $\left(\mathcal{C}_{1}\right)$;

- Technical capability $\left(\mathcal{C}_{2}\right)$;

- Experience level $\left(\mathcal{C}_{3}\right)$;

- Tender price $\left(\mathcal{C}_{4}\right)$;

- Safety and quality $\left(\mathcal{C}_{5}\right)$;

- Financial strength and stability $\left(\mathcal{C}_{6}\right)$;

- Completion time $\left(\mathcal{C}_{7}\right)$.

The tender price $\left(\mathcal{C}_{4}\right)$ and completion time $\left(\mathcal{C}_{7}\right)$ are non-beneficial criteria, and the other criteria are beneficial. To assess the importance of the criteria and appraise the performance values of alternatives with respect to each criterion, the decision-makers use the linguistic terms shown in Tab. 1 (Keshavarz Ghorabaee et al., 2014). The corresponding interval type-2 fuzzy sets of these linguistic terms are also defined in Tab. 1. The performance values of the alternatives on each criterion given by the decision-makers are presented in Tab. 2. The importance weights of the criteria determined by these decision-makers are represented in Tab. 3. The process of using the extended EDAS method with IT2FSs is illustrated as follows:

Step 1. The average decision matrix $X$ can be calculated based on the Tab. 1, Tab. 2 and Equations (11) and (12). Tab. 4 shows the IT2FSs related to the elements of $X$ matrix

Step 2. The matrix of the criteria weights is calculated based on Tab. 3 and Equations (13) and (14).

$$
W=\left[\begin{array}{lllllll}
\tilde{\tilde{w}}_{1} & \tilde{\tilde{w}}_{2} & \tilde{\tilde{w}}_{3} & \tilde{\tilde{w}}_{4} & \tilde{\tilde{w}}_{5} & \tilde{\tilde{w}}_{6} & \tilde{\tilde{w}}_{7}
\end{array}\right]
$$

The results are shown in Tab. 5 .

\section{Tab. 3: Weights of the criteria evaluated by the decision makers}

\begin{tabular}{c|c|c|c}
\multirow{2}{*}{ Criteria } & \multicolumn{3}{|c}{ Decision-makers } \\
\cline { 2 - 4 } & $\mathcal{D}_{1}$ & $\mathcal{D}_{2}$ & $\mathcal{D}_{3}$ \\
\hline $\mathcal{C}_{1}$ & $\mathrm{H}$ & $\mathrm{VH}$ & $\mathrm{VH}$ \\
\hline $\mathcal{C}_{2}$ & $\mathrm{MH}$ & $\mathrm{H}$ & $\mathrm{MH}$ \\
\hline $\mathcal{C}_{3}$ & $\mathrm{M}$ & $\mathrm{M}$ & $\mathrm{ML}$ \\
\hline $\mathcal{C}_{4}$ & $\mathrm{VH}$ & $\mathrm{VH}$ & $\mathrm{H}$ \\
\hline $\mathcal{C}_{5}$ & $\mathrm{H}$ & $\mathrm{H}$ & $\mathrm{MH}$ \\
\hline $\mathcal{C}_{6}$ & $\mathrm{M}$ & $\mathrm{MH}$ & $\mathrm{M}$ \\
\hline $\mathcal{C}_{7}$ & $\mathrm{MH}$ & $\mathrm{MH}$ & $\mathrm{M}$ \\
\hline
\end{tabular}

\section{Tab. 4: The elements of the average decision-matrix $(X)$ - Part 1}

\begin{tabular}{|c|c|c|c|c|c|c|c|c|c|c|c|c|}
\hline & \multicolumn{6}{|c|}{$\tilde{x}_{i j}^{U}$} & \multicolumn{6}{|c|}{$\tilde{x}_{i j}^{L}$} \\
\hline & $x_{1}^{U}$ & $x_{2}^{U}$ & $x_{3}^{U}$ & $x_{4}^{U}$ & $H_{I}^{U}$ & $H_{2}^{U}$ & $x_{1}^{L}$ & $x_{2}^{L}$ & $x_{3}^{L}$ & $x_{4}^{L}$ & $H_{1}^{L}$ & $H_{2}^{L}$ \\
\hline$\tilde{\tilde{x}}_{\mu l}$ & 0.37 & 0.57 & 0.62 & 0.77 & 1 & 1 & 0.47 & 0.57 & 0.62 & 0.67 & 0.9 & 0.9 \\
\hline$\tilde{\tilde{x}}_{21}$ & 0.07 & 0.23 & 0.28 & 0.43 & 1 & 1 & 0.15 & 0.23 & 0.28 & 0.33 & 0.9 & 0.9 \\
\hline$\tilde{\tilde{x}}_{31}$ & 0.03 & 0.13 & 0.17 & 0.30 & 1 & 1 & 0.08 & 0.13 & 0.17 & 0.22 & 0.9 & 0.9 \\
\hline$\tilde{\tilde{x}}_{41}$ & 0.63 & 0.80 & 0.85 & 0.97 & 1 & 1 & 0.73 & 0.80 & 0.85 & 0.90 & 0.9 & 0.9 \\
\hline$\tilde{\tilde{x}}_{51}$ & 0.77 & 0.90 & 0.93 & 1 & 1 & 1 & 0.85 & 0.90 & 0.93 & 0.97 & 0.9 & 0.9 \\
\hline$\tilde{\tilde{x}}_{61}$ & 0.90 & 1 & 1 & 1 & 1 & 1 & 0.95 & 1 & 1 & 1 & 0.9 & 0.9 \\
\hline$\tilde{\tilde{x}}_{71}$ & 0.37 & 0.57 & 0.62 & 0.77 & 1 & 1 & 0.47 & 0.57 & 0.62 & 0.67 & 0.9 & 0.9 \\
\hline
\end{tabular}




\section{Tab. 4: The elements of the average decision-matrix (X) - Part 2}

\begin{tabular}{|c|c|c|c|c|c|c|c|c|c|c|c|c|}
\hline & \multicolumn{6}{|c|}{$\tilde{x}_{i j}^{U}$} & \multicolumn{6}{|c|}{$\tilde{x}_{i j}^{L}$} \\
\hline & $x_{1}^{U}$ & $x_{2}^{U}$ & $x_{3}^{U}$ & $x_{4}^{U}$ & $H_{1}^{U}$ & $H_{2}^{U}$ & $x_{1}^{L}$ & $x_{2}^{L}$ & $x_{3}^{L}$ & $x_{4}^{L}$ & $H_{l}^{L}$ & $H_{2}^{L}$ \\
\hline$\tilde{\tilde{x}}_{81}$ & 0.50 & 0.68 & 0.73 & 0.87 & 1 & 1 & 0.60 & 0.68 & 0.73 & 0.78 & 0.9 & 0.9 \\
\hline$\tilde{\tilde{x}}_{12}$ & 0.17 & 0.37 & 0.42 & 0.57 & 1 & 1 & 0.27 & 0.37 & 0.42 & 0.47 & 0.9 & 0.9 \\
\hline$\tilde{\tilde{x}}_{22}$ & 0.57 & 0.75 & 0.80 & 0.93 & 1 & 1 & 0.67 & 0.75 & 0.80 & 0.85 & 0.9 & 0.9 \\
\hline$\tilde{\tilde{x}}_{32}$ & 0.70 & 0.85 & 0.88 & 0.97 & 1 & 1 & 0.78 & 0.85 & 0.88 & 0.92 & 0.9 & 0.9 \\
\hline$\tilde{\tilde{x}}_{42}$ & 0.83 & 0.95 & 0.97 & 1 & 1 & 1 & 0.90 & 0.95 & 0.97 & 0.98 & 0.9 & 0.9 \\
\hline$\tilde{\tilde{x}}_{52}$ & 0.43 & 0.63 & 0.68 & 0.83 & 1 & 1 & 0.53 & 0.63 & 0.68 & 0.73 & 0.9 & 0.9 \\
\hline$\tilde{\tilde{x}}_{62}$ & 0.70 & 0.85 & 0.90 & 1 & 1 & 1 & 0.80 & 0.85 & 0.90 & 0.95 & 0.9 & 0.9 \\
\hline$\tilde{\tilde{x}}_{72}$ & 0.43 & 0.63 & 0.68 & 0.83 & 1 & 1 & 0.53 & 0.63 & 0.68 & 0.73 & 0.9 & 0.9 \\
\hline$\tilde{\tilde{x}}_{82}$ & 0.03 & 0.13 & 0.17 & 0.30 & 1 & 1 & 0.08 & 0.13 & 0.17 & 0.22 & 0.9 & 0.9 \\
\hline$\tilde{\tilde{x}}_{13}$ & 0.17 & 0.37 & 0.42 & 0.57 & 1 & 1 & 0.27 & 0.37 & 0.42 & 0.47 & 0.9 & 0.9 \\
\hline$\tilde{\tilde{x}}_{23}$ & 0.43 & 0.63 & 0.68 & 0.83 & 1 & 1 & 0.53 & 0.63 & 0.68 & 0.73 & 0.9 & 0.9 \\
\hline$\tilde{\tilde{x}}_{33}$ & 0.37 & 0.57 & 0.62 & 0.77 & 1 & 1 & 0.47 & 0.57 & 0.62 & 0.67 & 0.9 & 0.9 \\
\hline$\tilde{\tilde{x}}_{43}$ & 0.77 & 0.90 & 0.93 & 1 & 1 & 1 & 0.85 & 0.90 & 0.93 & 0.97 & 0.9 & 0.9 \\
\hline$\tilde{\tilde{x}}_{53}$ & 0.83 & 0.95 & 0.97 & 1 & 1 & 1 & 0.90 & 0.95 & 0.97 & 0.98 & 0.9 & 0.9 \\
\hline$\tilde{\tilde{x}}_{63}$ & 0.63 & 0.80 & 0.85 & 0.97 & 1 & 1 & 0.73 & 0.80 & 0.85 & 0.90 & 0.9 & 0.9 \\
\hline$\tilde{\tilde{x}}_{73}$ & 0.70 & 0.85 & 0.88 & 0.97 & 1 & 1 & 0.78 & 0.85 & 0.88 & 0.92 & 0.9 & 0.9 \\
\hline$\tilde{\tilde{x}}_{83}$ & 0.03 & 0.17 & 0.22 & 0.37 & 1 & 1 & 0.10 & 0.17 & 0.22 & 0.27 & 0.9 & 0.9 \\
\hline$\tilde{\tilde{x}}_{14}$ & 0.23 & 0.43 & 0.48 & 0.63 & 1 & 1 & 0.33 & 0.43 & 0.48 & 0.53 & 0.9 & 0.9 \\
\hline$\tilde{\tilde{x}}_{24}$ & 0.70 & 0.85 & 0.88 & 0.97 & 1 & 1 & 0.78 & 0.85 & 0.88 & 0.92 & 0.9 & 0.9 \\
\hline$\tilde{\tilde{x}}_{34}$ & 0.43 & 0.63 & 0.68 & 0.83 & 1 & 1 & 0.53 & 0.63 & 0.68 & 0.73 & 0.9 & 0.9 \\
\hline$\tilde{\tilde{x}}_{44}$ & 0.00 & 0.10 & 0.15 & 0.30 & 1 & 1 & 0.05 & 0.10 & 0.15 & 0.20 & 0.9 & 0.9 \\
\hline$\tilde{\tilde{x}}_{54}$ & 0.00 & 0.03 & 0.05 & 0.17 & 1 & 1 & 0.02 & 0.03 & 0.05 & 0.10 & 0.9 & 0.9 \\
\hline$\tilde{\tilde{x}}_{64}$ & 0.03 & 0.13 & 0.17 & 0.30 & 1 & 1 & 0.08 & 0.13 & 0.17 & 0.22 & 0.9 & 0.9 \\
\hline$\tilde{\tilde{x}}_{74}$ & 0.50 & 0.68 & 0.73 & 0.87 & 1 & 1 & 0.60 & 0.68 & 0.73 & 0.78 & 0.9 & 0.9 \\
\hline$\tilde{\tilde{x}}_{84}$ & 0.43 & 0.63 & 0.68 & 0.83 & 1 & 1 & 0.53 & 0.63 & 0.68 & 0.73 & 0.9 & 0.9 \\
\hline$\tilde{\tilde{x}}_{15}$ & 0.57 & 0.75 & 0.80 & 0.93 & 1 & 1 & 0.67 & 0.75 & 0.80 & 0.85 & 0.9 & 0.9 \\
\hline$\tilde{\tilde{x}}_{25}$ & 0.83 & 0.95 & 0.97 & 1 & 1 & 1 & 0.90 & 0.95 & 0.97 & 0.98 & 0.9 & 0.9 \\
\hline$\tilde{\tilde{x}}_{35}$ & 0.57 & 0.75 & 0.80 & 0.93 & 1 & 1 & 0.67 & 0.75 & 0.80 & 0.85 & 0.9 & 0.9 \\
\hline$\tilde{\tilde{x}}_{45}$ & 0.70 & 0.85 & 0.90 & 1 & 1 & 1 & 0.80 & 0.85 & 0.90 & 0.95 & 0.9 & 0.9 \\
\hline$\tilde{\tilde{x}}_{55}$ & 0.50 & 0.68 & 0.73 & 0.87 & 1 & 1 & 0.60 & 0.68 & 0.73 & 0.78 & 0.9 & 0.9 \\
\hline$\tilde{\tilde{x}}_{65}$ & 0.90 & 1 & 1 & 1 & 1 & 1 & 0.95 & 1 & 1 & 1 & 0.9 & 0.9 \\
\hline$\tilde{\tilde{x}}_{75}$ & 0.23 & 0.43 & 0.48 & 0.63 & 1 & 1 & 0.33 & 0.43 & 0.48 & 0.53 & 0.9 & 0.9 \\
\hline$\tilde{\tilde{x}}_{85}$ & 0.03 & 0.13 & 0.17 & 0.30 & 1 & 1 & 0.08 & 0.13 & 0.17 & 0.22 & 0.9 & 0.9 \\
\hline$\tilde{\tilde{x}}_{16}$ & 0.13 & 0.30 & 0.35 & 0.50 & 1 & 1 & 0.22 & 0.30 & 0.35 & 0.40 & 0.9 & 0.9 \\
\hline$\tilde{\tilde{x}}_{26}$ & 0.63 & 0.80 & 0.85 & 0.97 & 1 & 1 & 0.73 & 0.80 & 0.85 & 0.90 & 0.9 & 0.9 \\
\hline
\end{tabular}




\section{Tab. 4: The elements of the average decision-matrix (X) - Part 3}

\begin{tabular}{c|c|c|c|c|c|c|c|c|c|c|c|c} 
& \multicolumn{7}{|c|}{$\tilde{x}^{U}$} \\
\cline { 2 - 15 } & $x_{I}^{U}$ & $x_{2}^{U}$ & $x_{3}^{U}$ & $x_{4}^{U}$ & $H_{I}^{U}$ & $H_{2}^{U}$ & $x_{I}^{L}$ & $x_{2}^{L}$ & $x_{3}^{L}$ & $x_{4}^{L}$ & $H_{I}^{L}$ & $H_{2}^{L}$ \\
\hline$\tilde{\tilde{x}}_{36}$ & 0.57 & 0.75 & 0.80 & 0.93 & 1 & 1 & 0.67 & 0.75 & 0.80 & 0.85 & 0.9 & 0.9 \\
\hline$\tilde{\tilde{x}}_{46}$ & 0.50 & 0.68 & 0.73 & 0.87 & 1 & 1 & 0.60 & 0.68 & 0.73 & 0.78 & 0.9 & 0.9 \\
\hline$\tilde{\tilde{x}}_{56}$ & 0.37 & 0.57 & 0.62 & 0.77 & 1 & 1 & 0.47 & 0.57 & 0.62 & 0.67 & 0.9 & 0.9 \\
\hline$\tilde{\tilde{x}}_{66}$ & 0.83 & 0.95 & 0.97 & 1 & 1 & 1 & 0.90 & 0.95 & 0.97 & 0.98 & 0.9 & 0.9 \\
\hline$\tilde{\tilde{x}}_{76}$ & 0.50 & 0.68 & 0.73 & 0.87 & 1 & 1 & 0.60 & 0.68 & 0.73 & 0.78 & 0.9 & 0.9 \\
\hline$\tilde{\tilde{x}}_{86}$ & 0.30 & 0.50 & 0.55 & 0.70 & 1 & 1 & 0.40 & 0.50 & 0.55 & 0.60 & 0.9 & 0.9 \\
\hline$\tilde{x}_{17}$ & 0.77 & 0.90 & 0.93 & 1 & 1 & 1 & 0.85 & 0.90 & 0.93 & 0.97 & 0.9 & 0.9 \\
\hline$\tilde{x}_{27}$ & 0.30 & 0.50 & 0.55 & 0.70 & 1 & 1 & 0.40 & 0.50 & 0.55 & 0.60 & 0.9 & 0.9 \\
\hline$\tilde{\tilde{x}}_{37}$ & 0.30 & 0.50 & 0.55 & 0.70 & 1 & 1 & 0.40 & 0.50 & 0.55 & 0.60 & 0.9 & 0.9 \\
\hline$\tilde{\tilde{x}}_{47}$ & 0.03 & 0.13 & 0.17 & 0.30 & 1 & 1 & 0.08 & 0.13 & 0.17 & 0.22 & 0.9 & 0.9 \\
\hline$\tilde{\tilde{x}}_{57}$ & 0.13 & 0.30 & 0.35 & 0.50 & 1 & 1 & 0.22 & 0.30 & 0.35 & 0.40 & 0.9 & 0.9 \\
\hline$\tilde{x}_{67}$ & 0.00 & 0.00 & 0.00 & 0.10 & 1 & 1 & 0.00 & 0.00 & 0.00 & 0.05 & 0.9 & 0.9 \\
\hline$\tilde{\tilde{x}}_{77}$ & 0.43 & 0.63 & 0.68 & 0.83 & 1 & 1 & 0.53 & 0.63 & 0.68 & 0.73 & 0.9 & 0.9 \\
\hline$\tilde{x}_{87}$ & 0.77 & 0.90 & 0.92 & 0.97 & 1 & 1 & 0.83 & 0.90 & 0.92 & 0.93 & 0.9 & 0.9 \\
\hline
\end{tabular}

\section{Tab. 5: The elements of the matrix of criteria weights}

\begin{tabular}{|c|c|c|c|c|c|c|c|c|c|c|c|c|}
\hline & \multicolumn{6}{|c|}{$\tilde{w}_{j}^{U}$} & \multicolumn{6}{|c|}{$\tilde{w}_{j}^{L}$} \\
\hline & $w_{1}^{U}$ & $w_{2}^{U}$ & $w_{3}^{U}$ & $w_{4}^{U}$ & $H_{I}^{U}$ & $H_{2}^{U}$ & $w_{1}^{L}$ & $w_{2}^{L}$ & $w_{3}^{L}$ & $w_{4}^{L}$ & $H_{I}^{L}$ & $H_{2}^{L}$ \\
\hline$\tilde{\tilde{w}}_{I}$ & 0.83 & 0.95 & 0.97 & 1 & 1 & 1 & 0.90 & 0.95 & 0.97 & 0.98 & 0.9 & 0.9 \\
\hline$\tilde{\tilde{w}}_{2}$ & 0.57 & 0.75 & 0.80 & 0.93 & 1 & 1 & 0.67 & 0.75 & 0.80 & 0.85 & 0.9 & 0.9 \\
\hline$\tilde{\tilde{w}}_{3}$ & 0.23 & 0.43 & 0.48 & 0.63 & 1 & 1 & 0.33 & 0.43 & 0.48 & 0.53 & 0.9 & 0.9 \\
\hline$\tilde{\tilde{w}}_{4}$ & 0.83 & 0.95 & 0.97 & 1 & 1 & 1 & 0.90 & 0.95 & 0.97 & 0.98 & 0.9 & 0.9 \\
\hline$\tilde{\tilde{w}}_{5}$ & 0.63 & 0.80 & 0.85 & 0.97 & 1 & 1 & 0.73 & 0.80 & 0.85 & 0.90 & 0.9 & 0.9 \\
\hline$\tilde{\tilde{w}}_{6}$ & 0.37 & 0.57 & 0.62 & 0.77 & 1 & 1 & 0.47 & 0.57 & 0.62 & 0.67 & 0.9 & 0.8 \\
\hline$\tilde{\tilde{w}}_{7}$ & 0.43 & 0.63 & 0.68 & 0.83 & 1 & 1 & 0.53 & 0.63 & 0.68 & 0.73 & 0.9 & 0.9 \\
\hline
\end{tabular}

Source: own

Step 3. The matrix of average solution can be calculated by using the results of Step 1 and Equations (15) and (16).

$$
A V=\left[\begin{array}{lllllll}
\widetilde{\widetilde{M}}_{1} & \widetilde{\widetilde{M}}_{2} & \widetilde{\widetilde{M}}_{3} & \widetilde{\widetilde{M}}_{4} & \widetilde{\widetilde{M}}_{5} & \widetilde{\widetilde{M}}_{6} & \widetilde{\tilde{M}}_{7}
\end{array}\right]
$$

The elements of the matrix of average solutions are represented in Tab. 6 .
Step 4. Based on Tab. 4 and Tab. 6 and Equations (17) to (20), the positive and negative distances (the PDA and NDA matrices) are calculated.

The positive distances are shown in Tab. 7 and the negative distances are represented in Tab. 8. 


\section{Tab. 6: The elements of the matrix of average solutions}

\begin{tabular}{|c|c|c|c|c|c|c|c|c|c|c|c|c|}
\hline & \multicolumn{6}{|c|}{$\tilde{\mathcal{M}}_{j}^{U}$} & \multicolumn{6}{|c|}{$\tilde{\mathcal{M}}_{j}^{L}$} \\
\hline & $\mathcal{M}_{I}^{U}$ & $\mathcal{M}_{2}^{U}$ & $\mathcal{M}_{3}^{U}$ & $\mathcal{M}_{4}^{U}$ & $H_{1}^{U}$ & $H_{2}^{U}$ & $\mathcal{M}_{1}^{L}$ & $\mathcal{M}_{2}^{L}$ & $\mathcal{M}_{3}^{L}$ & $\mathcal{M}_{4}^{L}$ & $H_{I}^{L}$ & $H_{2}^{L}$ \\
\hline$\widetilde{\tilde{M}}_{1}$ & 0.45 & 0.61 & 0.65 & 0.76 & 1 & 1 & 0.54 & 0.61 & 0.65 & 0.69 & 0.9 & 0.9 \\
\hline$\widetilde{\widetilde{M}}_{2}$ & 0.48 & 0.65 & 0.69 & 0.80 & 1 & 1 & 0.57 & 0.65 & 0.69 & 0.73 & 0.9 & 0.9 \\
\hline$\widetilde{\tilde{M}}_{3}$ & 0.49 & 0.65 & 0.70 & 0.81 & 1 & 1 & 0.58 & 0.65 & 0.70 & 0.74 & 0.9 & 0.9 \\
\hline$\widetilde{\tilde{M}}_{4}$ & 0.29 & 0.44 & 0.48 & 0.61 & 1 & 1 & 0.37 & 0.44 & 0.48 & 0.53 & 0.9 & 0.9 \\
\hline$\widetilde{\tilde{M}}_{5}$ & 0.54 & 0.69 & 0.73 & 0.83 & 1 & 1 & 0.63 & 0.69 & 0.73 & 0.77 & 0.9 & 0.9 \\
\hline$\widetilde{\tilde{M}}_{6}$ & 0.48 & 0.65 & 0.70 & 0.83 & 1 & 1 & 0.57 & 0.65 & 0.70 & 0.75 & 0.9 & 0.9 \\
\hline$\widetilde{\tilde{M}}_{7}$ & 0.34 & 0.48 & 0.52 & 0.64 & 1 & 1 & 0.41 & 0.48 & 0.52 & 0.56 & 0.9 & 0.9 \\
\hline
\end{tabular}

\section{Tab. 7: The positive distances from the average solution (PDA matrix) - Part 1}

\begin{tabular}{|c|c|c|c|c|c|c|c|c|c|c|c|c|}
\hline & \multicolumn{6}{|c|}{$\tilde{p}_{i j}^{U}$} & \multicolumn{6}{|c|}{$\tilde{p}_{i j}^{L}$} \\
\hline & $p_{1}^{U}$ & $p_{2}^{U}$ & $p_{3}^{U}$ & $p_{4}^{U}$ & $H_{I}^{U}$ & $H_{2}^{U}$ & $p_{I}^{L}$ & $p_{2}^{L}$ & $p_{3}^{L}$ & $p_{4}^{L}$ & $H_{I}^{L}$ & $H_{2}^{L}$ \\
\hline$\tilde{\tilde{p}}_{11}$ & 0 & 0 & 0 & 0 & 1 & 1 & 0 & 0 & 0 & 0 & 1 & 1 \\
\hline$\tilde{\tilde{p}}_{21}$ & 0 & 0 & 0 & 0 & 1 & 1 & 0 & 0 & 0 & 0 & 1 & 1 \\
\hline$\tilde{\tilde{p}}_{31}$ & 0 & 0 & 0 & 0 & 1 & 1 & 0 & 0 & 0 & 0 & 1 & 1 \\
\hline$\tilde{\tilde{p}}_{41}$ & -0.21 & 0.24 & 0.38 & 0.82 & 1 & 1 & 0.07 & 0.24 & 0.38 & 0.58 & 0.9 & 0.9 \\
\hline$\tilde{\tilde{p}}_{51}$ & 0.01 & 0.40 & 0.52 & 0.87 & 1 & 1 & 0.25 & 0.40 & 0.52 & 0.69 & 0.9 & 0.9 \\
\hline$\tilde{\tilde{p}}_{61}$ & 0.22 & 0.56 & 0.62 & 0.87 & 1 & 1 & 0.41 & 0.56 & 0.62 & 0.74 & 0.9 & 0.9 \\
\hline$\tilde{\tilde{p}}_{71}$ & 0 & 0 & 0 & 0 & 1 & 1 & 0 & 0 & 0 & 0 & 1 & 1 \\
\hline$\tilde{\tilde{p}}_{81}$ & -0.42 & 0.05 & 0.20 & 0.66 & 1 & 1 & -0.15 & 0.05 & 0.20 & 0.39 & 0.9 & 0.9 \\
\hline$\tilde{\tilde{p}}_{12}$ & 0 & 0 & 0 & 0 & 1 & 1 & 0 & 0 & 0 & 0 & 1 & 1 \\
\hline$\tilde{\tilde{p}}_{22}$ & -0.36 & 0.09 & 0.23 & 0.68 & 1 & 1 & -0.10 & 0.09 & 0.23 & 0.42 & 0.9 & 0.9 \\
\hline$\tilde{\tilde{\mathcal{p}}}_{32}$ & -0.16 & 0.25 & 0.36 & 0.73 & 1 & 1 & 0.08 & 0.25 & 0.36 & 0.52 & 0.9 & 0.9 \\
\hline$\tilde{\tilde{p}}_{42}$ & 0.04 & 0.40 & 0.49 & 0.78 & 1 & 1 & 0.26 & 0.40 & 0.49 & 0.62 & 0.9 & 0.9 \\
\hline$\tilde{\tilde{p}}_{52}$ & 0 & 0 & 0 & 0 & 1 & 1 & 0 & 0 & 0 & 0 & 1 & 1 \\
\hline$\tilde{\tilde{p}}_{62}$ & -0.16 & 0.25 & 0.39 & 0.78 & 1 & 1 & 0.10 & 0.25 & 0.39 & 0.57 & 0.9 & 0.9 \\
\hline$\tilde{\tilde{p}}_{72}$ & 0 & 0 & 0 & 0 & 1 & 1 & 0 & 0 & 0 & 0 & 1 & 1 \\
\hline
\end{tabular}




\section{Tab. 7: The positive distances from the average solution (PDA matrix) - Part 2}

\begin{tabular}{|c|c|c|c|c|c|c|c|c|c|c|c|c|}
\hline & \multicolumn{6}{|c|}{$\tilde{p}_{i j}^{U}$} & \multicolumn{6}{|c|}{$\tilde{p}_{i j}^{L}$} \\
\hline & $p_{I}^{U}$ & $p_{2}^{U}$ & $p_{3}^{U}$ & $p_{4}^{U}$ & $H_{I}^{U}$ & $H_{2}^{U}$ & $p_{1}^{L}$ & $p_{2}^{L}$ & $p_{3}^{L}$ & $p_{4}^{L}$ & $H_{I}^{L}$ & $H_{2}^{L}$ \\
\hline$\tilde{\tilde{p}}_{82}$ & 0 & 0 & 0 & 0 & 1 & 1 & 0 & 0 & 0 & 0 & 1 & 1 \\
\hline$\tilde{\tilde{p}}_{13}$ & 0 & 0 & 0 & 0 & 1 & 1 & 0 & 0 & 0 & 0 & 1 & 1 \\
\hline$\tilde{\tilde{p}}_{23}$ & 0 & 0 & 0 & 0 & 1 & 1 & 0 & 0 & 0 & 0 & 1 & 1 \\
\hline$\tilde{\tilde{p}}_{33}$ & 0 & 0 & 0 & 0 & 1 & 1 & 0 & 0 & 0 & 0 & 1 & 1 \\
\hline$\tilde{\tilde{p}}_{43}$ & -0.06 & 0.31 & 0.42 & 0.76 & 1 & 1 & 0.17 & 0.31 & 0.42 & 0.58 & 0.9 & 0.9 \\
\hline$\tilde{\tilde{p}}_{53}$ & 0.04 & 0.38 & 0.47 & 0.76 & 1 & 1 & 0.24 & 0.38 & 0.47 & 0.61 & 0.9 & 0.9 \\
\hline$\tilde{\tilde{p}}_{63}$ & -0.26 & 0.16 & 0.29 & 0.71 & 1 & 1 & -0.01 & 0.16 & 0.29 & 0.48 & 0.9 & 0.9 \\
\hline$\tilde{\tilde{p}}_{73}$ & -0.16 & 0.23 & 0.34 & 0.71 & 1 & 1 & 0.07 & 0.23 & 0.34 & 0.51 & 0.9 & 0.9 \\
\hline$\tilde{\tilde{p}}_{83}$ & 0 & 0 & 0 & 0 & 1 & 1 & 0 & 0 & 0 & 0 & 1 & 1 \\
\hline$\tilde{\tilde{p}}_{14}$ & -0.75 & -0.10 & 0.10 & 0.83 & 1 & 1 & -0.37 & -0.10 & 0.10 & 0.43 & 0.9 & 0.9 \\
\hline$\tilde{\tilde{p}}_{24}$ & 0 & 0 & 0 & 0 & 1 & 1 & 0 & 0 & 0 & 0 & 1 & 1 \\
\hline$\tilde{\tilde{p}}_{34}$ & 0 & 0 & 0 & 0 & 1 & 1 & 0 & 0 & 0 & 0 & 1 & 1 \\
\hline$\tilde{\tilde{p}}_{44}$ & -0.02 & 0.63 & 0.83 & 1.35 & 1 & 1 & 0.37 & 0.63 & 0.83 & 1.05 & 0.9 & 0.9 \\
\hline$\tilde{\tilde{p}}_{54}$ & 0.27 & 0.85 & 0.98 & 1.35 & 1 & 1 & 0.59 & 0.85 & 0.98 & 1.12 & 0.9 & 0.9 \\
\hline$\tilde{\tilde{p}}_{64}$ & -0.02 & 0.59 & 0.76 & 1.27 & 1 & 1 & 0.33 & 0.59 & 0.76 & 0.97 & 0.9 & 0.9 \\
\hline$\tilde{\tilde{p}}_{74}$ & 0 & 0 & 0 & 0 & 1 & 1 & 0 & 0 & 0 & 0 & 1 & 1 \\
\hline$\tilde{\tilde{p}}_{84}$ & 0 & 0 & 0 & 0 & 1 & 1 & 0 & 0 & 0 & 0 & 1 & 1 \\
\hline$\tilde{\tilde{p}}_{15}$ & -0.38 & 0.03 & 0.15 & 0.55 & 1 & 1 & -0.15 & 0.03 & 0.15 & 0.32 & 0.9 & 0.9 \\
\hline$\tilde{\tilde{p}}_{25}$ & 0 & 0.31 & 0.39 & 0.65 & 1 & 1 & 0.18 & 0.31 & 0.39 & 0.51 & 0.9 & 0.9 \\
\hline$\tilde{\tilde{p}}_{35}$ & -0.38 & 0.03 & 0.15 & 0.55 & 1 & 1 & -0.15 & 0.03 & 0.15 & 0.32 & 0.9 & 0.9 \\
\hline$\tilde{\tilde{p}}_{45}$ & -0.19 & 0.17 & 0.29 & 0.65 & 1 & 1 & 0.04 & 0.17 & 0.29 & 0.46 & 0.9 & 0.9 \\
\hline$\tilde{\tilde{p}}_{55}$ & 0 & 0 & 0 & 0 & 1 & 1 & 0 & 0 & 0 & 0 & 1 & 1 \\
\hline$\tilde{\tilde{p}}_{65}$ & 0.09 & 0.38 & 0.43 & 0.65 & 1 & 1 & 0.25 & 0.38 & 0.43 & 0.53 & 0.9 & 0.9 \\
\hline$\tilde{\tilde{p}}_{75}$ & 0 & 0 & 0 & 0 & 1 & 1 & 0 & 0 & 0 & 0 & 1 & 1 \\
\hline$\tilde{\tilde{p}}_{85}$ & 0 & 0 & 0 & 0 & 1 & 1 & 0 & 0 & 0 & 0 & 1 & 1 \\
\hline$\tilde{\tilde{p}}_{16}$ & 0 & 0 & 0 & 0 & 1 & 1 & 0 & 0 & 0 & 0 & 1 & 1 \\
\hline$\tilde{\tilde{p}}_{26}$ & -0.29 & 0.15 & 0.29 & 0.73 & 1 & 1 & -0.02 & 0.15 & 0.29 & 0.49 & 0.9 & 0.9 \\
\hline
\end{tabular}




\section{Tab. 7: The positive distances from the average solution (PDA matrix) - Part 3}

\begin{tabular}{c|c|c|c|c|c|c|c|c|c|c|c|c} 
& \multicolumn{1}{|c}{$\tilde{p}_{i j}^{U}$} \\
\cline { 2 - 12 } & $p_{1}^{U}$ & $p_{2}^{U}$ & $p_{3}^{U}$ & $p_{4}^{U}$ & $H_{1}^{U}$ & $H_{2}^{U}$ & $p_{1}^{L}$ & $p_{2}^{L}$ & $p_{3}^{L}$ & $p_{4}^{L}$ & $H_{I}^{L}$ & $H_{2}^{L}$ \\
\hline$\tilde{\tilde{p}}_{36}$ & -0.39 & 0.07 & 0.22 & 0.68 & 1 & 1 & -0.12 & 0.07 & 0.22 & 0.41 & 0.9 & 0.9 \\
\hline$\tilde{\tilde{p}}_{46}$ & -0.49 & -0.02 & 0.12 & 0.58 & 1 & 1 & -0.22 & -0.02 & 0.12 & 0.31 & 0.9 & 0.9 \\
\hline$\tilde{\tilde{p}}_{56}$ & 0 & 0 & 0 & 0 & 1 & 1 & 0 & 0 & 0 & 0 & 1 & 1 \\
\hline$\tilde{\tilde{p}}_{66}$ & 0.01 & 0.37 & 0.47 & 0.78 & 1 & 1 & 0.23 & 0.37 & 0.47 & 0.61 & 0.9 & 0.9 \\
\hline$\tilde{\tilde{p}}_{76}$ & -0.49 & -0.02 & 0.12 & 0.58 & 1 & 1 & -0.22 & -0.02 & 0.12 & 0.31 & 0.9 & 0.9 \\
\hline$\tilde{\tilde{p}}_{86}$ & 0 & 0 & 0 & 0 & 1 & 1 & 0 & 0 & 0 & 0 & 1 & 1 \\
\hline$\tilde{\tilde{p}}_{17}$ & 0 & 0 & 0 & 0 & 1 & 1 & 0 & 0 & 0 & 0 & 1 & 1 \\
\hline$\tilde{\tilde{p}}_{27}$ & 0 & 0 & 0 & 0 & 1 & 1 & 0 & 0 & 0 & 0 & 1 & 1 \\
\hline$\tilde{\tilde{p}}_{37}$ & 0 & 0 & 0 & 0 & 1 & 1 & 0 & 0 & 0 & 0 & 1 & 1 \\
\hline$\tilde{\tilde{p}}_{47}$ & 0.08 & 0.64 & 0.78 & 1.22 & 1 & 1 & 0.40 & 0.64 & 0.78 & 0.96 & 0.9 & 0.9 \\
\hline$\tilde{\tilde{p}}_{57}$ & -0.32 & 0.27 & 0.44 & 1.01 & 1 & 1 & 0.03 & 0.27 & 0.44 & 0.70 & 0.9 & 0.9 \\
\hline$\tilde{\tilde{p}}_{67}$ & 0.49 & 0.97 & 1.04 & 1.28 & 1 & 1 & 0.73 & 0.97 & 1.04 & 1.13 & 0.9 & 0.9 \\
\hline$\tilde{\tilde{p}}_{77}$ & 0 & 0 & 0 & 0 & 1 & 1 & 0 & 0 & 0 & 0 & 1 & 1 \\
\hline$\tilde{\tilde{p}}_{87}$ & 0 & 0 & 0 & 0 & 1 & 1 & 0 & 0 & 0 & 0 & 1 & 1 \\
\hline
\end{tabular}

\section{Tab. 8: The negative distances from the average solution (NDA matrix) - Part 1}

\begin{tabular}{c|c|c|c|c|c|c|c|c|c|c|c|c} 
& \multicolumn{9}{|c|}{$\tilde{n}_{i j}^{U}$} & \multicolumn{7}{|c}{$\tilde{n}_{i j}^{L}$} \\
\cline { 2 - 15 } & $n_{1}^{U}$ & $n_{2}^{U}$ & $n_{3}^{U}$ & $n_{4}^{U}$ & $H_{1}^{U}$ & $H_{2}^{U}$ & $n_{1}^{L}$ & $n_{2}^{L}$ & $n_{3}^{L}$ & $n_{4}^{L}$ & $H_{1}^{L}$ & $H_{2}^{L}$ \\
\hline$\tilde{\tilde{n}}_{11}$ & -0.50 & -0.01 & 0.13 & 0.63 & 1 & 1 & -0.21 & -0.01 & 0.13 & 0.36 & 0.9 & 0.9 \\
\hline$\tilde{\tilde{n}}_{21}$ & 0.03 & 0.52 & 0.67 & 1.12 & 1 & 1 & 0.33 & 0.52 & 0.67 & 0.87 & 0.9 & 0.9 \\
\hline$\tilde{\tilde{n}}_{31}$ & 0.25 & 0.71 & 0.83 & 1.17 & 1 & 1 & 0.51 & 0.71 & 0.83 & 0.98 & 0.9 & 0.9 \\
\hline$\tilde{\tilde{n}}_{41}$ & 0 & 0 & 0 & 0 & 1 & 1 & 0 & 0 & 0 & 0 & 1 & 1 \\
\hline$\tilde{\tilde{n}}_{51}$ & 0 & 0 & 0 & 0 & 1 & 1 & 0 & 0 & 0 & 0 & 1 & 1 \\
\hline$\tilde{\tilde{n}}_{61}$ & 0 & 0 & 0 & 0 & 1 & 1 & 0 & 0 & 0 & 0 & 1 & 1 \\
\hline$\tilde{\tilde{n}}_{71}$ & -0.50 & -0.01 & 0.13 & 0.63 & 1 & 1 & -0.21 & -0.01 & 0.13 & 0.36 & 0.9 & 0.9 \\
\hline$\tilde{\tilde{n}}_{81}$ & 0 & 0 & 0 & 0 & 1 & 1 & 0 & 0 & 0 & 0 & 1 & 1 \\
\hline$\tilde{\tilde{n}}_{12}$ & -0.13 & 0.35 & 0.49 & 0.97 & 1 & 1 & 0.16 & 0.35 & 0.49 & 0.70 & 0.9 & 0.9 \\
\hline
\end{tabular}




\section{Tab. 8: The negative distances from the average solution (NDA matrix) - Part 2}

\begin{tabular}{|c|c|c|c|c|c|c|c|c|c|c|c|c|}
\hline & \multicolumn{6}{|c|}{$\tilde{n}_{i j}^{U}$} & \multicolumn{6}{|c|}{$\tilde{n}_{i j}^{L}$} \\
\hline & $n_{1}^{U}$ & $n_{2}^{U}$ & $n_{3}^{U}$ & $n_{4}^{U}$ & $H_{I}^{U}$ & $H_{2}^{U}$ & $n_{l}^{L}$ & $n_{2}^{L}$ & $n_{3}^{L}$ & $n_{4}^{L}$ & $H_{1}^{L}$ & $H_{2}^{L}$ \\
\hline$\tilde{\tilde{n}}_{22}$ & 0 & 0 & 0 & 0 & 1 & 1 & 0 & 0 & 0 & 0 & 1 & 1 \\
\hline$\tilde{\tilde{n}}_{32}$ & 0 & 0 & 0 & 0 & 1 & 1 & 0 & 0 & 0 & 0 & 1 & 1 \\
\hline$\tilde{\tilde{n}}_{42}$ & 0 & 0 & 0 & 0 & 1 & 1 & 0 & 0 & 0 & 0 & 1 & 1 \\
\hline$\tilde{\tilde{n}}_{52}$ & -0.53 & -0.06 & 0.08 & 0.56 & 1 & 1 & -0.25 & -0.06 & 0.08 & 0.30 & 0.9 & 0.9 \\
\hline$\tilde{\tilde{n}}_{62}$ & 0 & 0 & 0 & 0 & 1 & 1 & 0 & 0 & 0 & 0 & 1 & 1 \\
\hline$\tilde{\tilde{n}}_{72}$ & -0.53 & -0.06 & 0.08 & 0.56 & 1 & 1 & -0.25 & -0.06 & 0.08 & 0.30 & 0.9 & 0.9 \\
\hline$\tilde{\tilde{n}}_{82}$ & 0.28 & 0.73 & 0.84 & 1.17 & 1 & 1 & 0.54 & 0.73 & 0.84 & 0.98 & 0.9 & 0.9 \\
\hline$\tilde{\tilde{n}}_{13}$ & -0.11 & 0.36 & 0.49 & 0.96 & 1 & 1 & 0.17 & 0.36 & 0.49 & 0.70 & 0.9 & 0.9 \\
\hline$\tilde{\tilde{n}}_{23}$ & -0.51 & -0.04 & 0.09 & 0.56 & 1 & 1 & -0.23 & -0.04 & 0.09 & 0.31 & 0.9 & 0.9 \\
\hline$\tilde{\tilde{n}}_{33}$ & -0.41 & 0.06 & 0.19 & 0.66 & 1 & 1 & -0.13 & 0.06 & 0.19 & 0.41 & 0.9 & 0.9 \\
\hline$\tilde{\tilde{n}}_{43}$ & 0 & 0 & 0 & 0 & 1 & 1 & 0 & 0 & 0 & 0 & 1 & 1 \\
\hline$\tilde{\tilde{n}}_{53}$ & 0 & 0 & 0 & 0 & 1 & 1 & 0 & 0 & 0 & 0 & 1 & 1 \\
\hline$\tilde{\tilde{n}}_{63}$ & 0 & 0 & 0 & 0 & 1 & 1 & 0 & 0 & 0 & 0 & 1 & 1 \\
\hline$\tilde{\tilde{n}}_{73}$ & 0 & 0 & 0 & 0 & 1 & 1 & 0 & 0 & 0 & 0 & 1 & 1 \\
\hline$\tilde{\tilde{n}}_{83}$ & 0.19 & 0.65 & 0.79 & 1.16 & 1 & 1 & 0.47 & 0.65 & 0.79 & 0.95 & 0.9 & 0.9 \\
\hline$\tilde{\tilde{n}}_{14}$ & 0 & 0 & 0 & 0 & 1 & 1 & 0 & 0 & 0 & 0 & 1 & 1 \\
\hline$\tilde{\tilde{n}}_{24}$ & 0.19 & 0.81 & 0.98 & 1.48 & 1 & 1 & 0.56 & 0.81 & 0.98 & 1.21 & 0.9 & 0.9 \\
\hline$\tilde{\tilde{n}}_{34}$ & -0.39 & 0.34 & 0.54 & 1.19 & 1 & 1 & 0.01 & 0.34 & 0.54 & 0.81 & 0.9 & 0.9 \\
\hline$\tilde{\tilde{n}}_{44}$ & 0 & 0 & 0 & 0 & 1 & 1 & 0 & 0 & 0 & 0 & 1 & 1 \\
\hline$\tilde{\tilde{n}}_{54}$ & 0 & 0 & 0 & 0 & 1 & 1 & 0 & 0 & 0 & 0 & 1 & 1 \\
\hline$\tilde{\tilde{n}}_{64}$ & 0 & 0 & 0 & 0 & 1 & 1 & 0 & 0 & 0 & 0 & 1 & 1 \\
\hline$\tilde{\tilde{n}}_{74}$ & -0.25 & 0.45 & 0.65 & 1.26 & 1 & 1 & 0.16 & 0.45 & 0.65 & 0.92 & 0.9 & 0.9 \\
\hline$\tilde{\tilde{n}}_{84}$ & -0.39 & 0.34 & 0.54 & 1.19 & 1 & 1 & 0.01 & 0.34 & 0.54 & 0.81 & 0.9 & 0.9 \\
\hline$\tilde{\tilde{n}}_{15}$ & 0 & 0 & 0 & 0 & 1 & 1 & 0 & 0 & 0 & 0 & 1 & 1 \\
\hline$\tilde{\tilde{n}}_{25}$ & 0 & 0 & 0 & 0 & 1 & 1 & 0 & 0 & 0 & 0 & 1 & 1 \\
\hline$\tilde{\tilde{n}}_{35}$ & 0 & 0 & 0 & 0 & 1 & 1 & 0 & 0 & 0 & 0 & 1 & 1 \\
\hline$\tilde{\tilde{n}}_{45}$ & 0 & 0 & 0 & 0 & 1 & 1 & 0 & 0 & 0 & 0 & 1 & 1 \\
\hline$\tilde{\tilde{n}}_{55}$ & -0.46 & -0.06 & 0.07 & 0.47 & 1 & 1 & -0.22 & -0.06 & 0.07 & 0.24 & 0.9 & 0.9 \\
\hline$\tilde{\tilde{n}}_{65}$ & 0 & 0 & 0 & 0 & 1 & 1 & 0 & 0 & 0 & 0 & 1 & 1 \\
\hline$\tilde{\tilde{n}}_{75}$ & -0.13 & 0.30 & 0.42 & 0.85 & 1 & 1 & 0.13 & 0.30 & 0.42 & 0.62 & 0.9 & 0.9 \\
\hline
\end{tabular}


Tab. 8: The negative distances from the average solution (NDA matrix) - Part 3

\begin{tabular}{|c|c|c|c|c|c|c|c|c|c|c|c|c|}
\hline & \multicolumn{6}{|c|}{$\tilde{n}_{i j}^{U}$} & \multicolumn{6}{|c|}{$\tilde{n}_{i j}^{L}$} \\
\hline & $n_{1}^{U}$ & $n_{2}^{U}$ & $n_{3}^{U}$ & $n_{4}^{U}$ & $H_{1}^{U}$ & $H_{2}^{U}$ & $n_{1}^{L}$ & $n_{2}^{L}$ & $n_{3}^{L}$ & $n_{4}^{L}$ & $H_{I}^{L}$ & $H_{2}^{L}$ \\
\hline$\tilde{\tilde{n}}_{85}$ & 0.34 & 0.75 & 0.85 & 1.13 & 1 & 1 & 0.58 & 0.75 & 0.85 & 0.97 & 0.9 & 0.9 \\
\hline$\tilde{\tilde{n}}_{16}$ & -0.03 & 0.45 & 0.60 & 1.03 & 1 & 1 & 0.26 & 0.45 & 0.60 & 0.79 & 0.9 & 0.9 \\
\hline$\tilde{\tilde{n}}_{26}$ & 0 & 0 & 0 & 0 & 1 & 1 & 0 & 0 & 0 & 0 & 1 & 1 \\
\hline$\tilde{\tilde{n}}_{36}$ & 0 & 0 & 0 & 0 & 1 & 1 & 0 & 0 & 0 & 0 & 1 & 1 \\
\hline$\tilde{\tilde{n}}_{46}$ & 0 & 0 & 0 & 0 & 1 & 1 & 0 & 0 & 0 & 0 & 1 & 1 \\
\hline$\tilde{\tilde{n}}_{56}$ & -0.43 & 0.06 & 0.20 & 0.68 & 1 & 1 & -0.14 & 0.06 & 0.20 & 0.42 & 0.9 & 0.9 \\
\hline$\tilde{\tilde{n}}_{66}$ & 0 & 0 & 0 & 0 & 1 & 1 & 0 & 0 & 0 & 0 & 1 & 1 \\
\hline$\tilde{\tilde{n}}_{76}$ & 0 & 0 & 0 & 0 & 1 & 1 & 0 & 0 & 0 & 0 & 1 & 1 \\
\hline$\tilde{\tilde{n}}_{86}$ & -0.33 & 0.16 & 0.30 & 0.78 & 1 & 1 & -0.04 & 0.16 & 0.30 & 0.52 & 0.9 & 0.9 \\
\hline$\tilde{\tilde{n}}_{17}$ & 0.26 & 0.77 & 0.91 & 1.32 & 1 & 1 & 0.58 & 0.77 & 0.91 & 1.11 & 0.9 & 0.9 \\
\hline$\tilde{\tilde{n}}_{27}$ & -0.68 & -0.04 & 0.13 & 0.72 & 1 & 1 & -0.33 & -0.04 & 0.13 & 0.37 & 0.9 & 0.9 \\
\hline$\tilde{\tilde{n}}_{37}$ & -0.68 & -0.04 & 0.13 & 0.72 & 1 & 1 & -0.33 & -0.04 & 0.13 & 0.37 & 0.9 & 0.9 \\
\hline$\tilde{\tilde{n}}_{47}$ & 0 & 0 & 0 & 0 & 1 & 1 & 0 & 0 & 0 & 0 & 1 & 1 \\
\hline$\tilde{\tilde{n}}_{57}$ & 0 & 0 & 0 & 0 & 1 & 1 & 0 & 0 & 0 & 0 & 1 & 1 \\
\hline$\tilde{\tilde{n}}_{67}$ & 0 & 0 & 0 & 0 & 1 & 1 & 0 & 0 & 0 & 0 & 1 & 1 \\
\hline$\tilde{\tilde{n}}_{77}$ & -0.41 & 0.23 & 0.40 & 0.99 & 1 & 1 & -0.06 & 0.23 & 0.40 & 0.64 & 0.9 & 0.9 \\
\hline$\tilde{\tilde{n}}_{87}$ & 0.26 & 0.77 & 0.87 & 1.26 & 1 & 1 & 0.54 & 0.77 & 0.87 & 1.04 & 0.9 & 0.9 \\
\hline
\end{tabular}

Source: own

A reciprocal relation between corresponding elements of PDA and NDA matrices can be seen in Tab. 7 and Tab. 8. If an element of one of these matrices is equal to $\tilde{0}$, the corresponding element of the other matrix is a positive IT2FS, and vice versa.

Steps 5 to 7. Based on the Tab. 7 and Tab. 8 and Equations (21) to (25), the weighted sum of positive and negative distances $\left(\widetilde{\widetilde{s p}}_{i}\right.$ and $\left.\widetilde{\widetilde{s n}}_{i}\right)$, the normalized values of them $\left(\widetilde{\widetilde{n p}}_{i}\right.$ and $\widetilde{\widetilde{n n}}_{i}$ ) and the appraisal scores are calculated for all alternatives. The results of these steps are shown in Tab. 9.

Step 8. According to Tab. 9, the ranking values of appraisal scores $(\mathcal{R V})$ can be calculated. The results are represented in Tab. 10. It can be seen that the ranking order of alternatives (subcontractors) is $\mathcal{A}_{6}>\mathcal{A}_{4}>\mathcal{A}_{5}$ $>\mathcal{A}_{3}>\mathcal{A}_{2}>\mathcal{A}_{7}>\mathcal{A}_{1}>\mathcal{A}_{8}$. Therefore, $\mathcal{A}_{6}$ is the best subcontractor in terms of the seven criteria.

\section{Comparison and Sensitivity Analysis}

A comparison and a sensitivity analysis are performed in this section to validate the results of the proposed method. In recent years, many multi-criteria decision making methods have been introduced and developed in the interval type-2 fuzzy environment. Some methods which have good efficiency and could be implemented in the considered MCDM problem have been selected for the comparison. The selected methods for the comparative analysis are the methods proposed by Chen et al. (2012), Wang et al. (2012), Baležentis and Zeng (2013), Hu et al. (2013), Keshavarz Ghorabaee et al. (2014) 


\begin{tabular}{|c|c|c|c|c|c|c|c|c|c|c|c|c|c|}
\hline Tab & \multicolumn{13}{|c|}{$\begin{array}{l}\text { The weighted sum of distances, their normalized values and the appraisal } \\
\text { scores - Part } 1\end{array}$} \\
\hline \multirow{2}{*}{$\tilde{\tilde{K}}$} & & \multicolumn{6}{|c|}{$\tilde{K}_{i j}^{U}$} & \multicolumn{6}{|c|}{$\tilde{K}_{i j}^{L}$} \\
\hline & & $k_{1}^{U}$ & $k_{2}^{U}$ & $k_{3}^{U}$ & $k_{4}^{U}$ & $H_{I}^{U}$ & $H_{2}^{c}$ & $k_{I}^{L}$ & $k_{2}^{L}$ & $k_{3}^{L}$ & $k_{4}^{L}$ & $H_{I}^{L}$ & $H_{2}^{L}$ \\
\hline \multirow{8}{*}{$11 \infty$} & $\widetilde{\widetilde{s p}}_{1}$ & -1.12 & -0.08 & 0.23 & 1.37 & 1 & 1 & -0.49 & -0.08 & 0.23 & 0.71 & 0.9 & 0.9 \\
\hline & $\widetilde{\widetilde{s p}}_{2}$ & -0.56 & 0.40 & 0.70 & 1.82 & 1 & 1 & 0.04 & 0.40 & 0.70 & 1.14 & 0.9 & 0.9 \\
\hline & $\widetilde{\widetilde{s p}}_{3}$ & -0.81 & 0.25 & 0.55 & 1.74 & 1 & 1 & -0.16 & 0.25 & 0.55 & 1.01 & 0.9 & 0.9 \\
\hline & $\widetilde{\widetilde{s p}}_{4}$ & -0.76 & 1.78 & 2.62 & 5.46 & 1 & 1 & 0.71 & 1.78 & 2.62 & 3.77 & 0.9 & 0.9 \\
\hline & $\widetilde{\widetilde{s p}}_{5}$ & -0.02 & 1.52 & 1.97 & 3.55 & 1 & 1 & 0.85 & 1.52 & 1.97 & 2.61 & 0.9 & 0.9 \\
\hline & $\widetilde{\widetilde{s p}}_{6}$ & 0.13 & 2.48 & 3.16 & 5.62 & 1 & 1 & 1.42 & 2.48 & 3.16 & 4.15 & 0.9 & 0.9 \\
\hline & $\widetilde{s p}_{7}$ & -0.47 & 0.08 & 0.24 & 0.89 & 1 & 1 & -0.12 & 0.08 & 0.24 & 0.48 & 0.9 & 0.9 \\
\hline & ${\widetilde{s p_{8}}}$ & -0.42 & 0.05 & 0.19 & 0.66 & 1 & 1 & -0.14 & 0.05 & 0.19 & 0.39 & 0.9 & 0.9 \\
\hline \multirow{8}{*}{$11 \underbrace{\infty}_{\infty}$} & $\widetilde{\widetilde{s n}}_{1}$ & -0.60 & 1.15 & 1.74 & 4.04 & 1 & 1 & 0.39 & 1.15 & 1.74 & 2.67 & 0.9 & 0.9 \\
\hline & $\widetilde{\widetilde{s n}}_{2}$ & -0.70 & 1.22 & 1.73 & 3.55 & 1 & 1 & 0.44 & 1.22 & 1.73 & 2.48 & 0.9 & 0.9 \\
\hline & $\widetilde{8 n}_{3}$ & -1.01 & 1.00 & 1.51 & 3.38 & 1 & 1 & 0.17 & 1.00 & 1.51 & 2.24 & 0.9 & 0.9 \\
\hline & $\widetilde{\widetilde{8 n}}_{4}$ & 0.00 & 0.00 & 0.00 & 0.00 & 1 & 1 & 0.00 & 0.00 & 0.00 & 0.00 & 0.9 & 0.9 \\
\hline & $\widetilde{\widetilde{s n}}_{5}$ & -1.27 & -0.06 & 0.25 & 1.51 & 1 & 1 & -0.50 & -0.06 & 0.25 & 0.75 & 0.9 & 0.9 \\
\hline & $\widetilde{\widetilde{s n}}_{6}$ & 0.00 & 0.00 & 0.00 & 0.00 & 1 & 1 & 0.00 & 0.00 & 0.00 & 0.00 & 0.9 & 0.9 \\
\hline & $\widetilde{s n}_{7}$ & -1.71 & 0.76 & 1.46 & 4.07 & 1 & 1 & -0.22 & 0.76 & 1.46 & 2.54 & 0.9 & 0.9 \\
\hline & $\widetilde{\widetilde{s n}}_{8}$ & -0.12 & 2.32 & 3.08 & 5.76 & 1 & 1 & 1.21 & 2.32 & 3.08 & 4.12 & 0.9 & 0.9 \\
\hline \multirow{8}{*}{$\|$} & $\widetilde{\widetilde{n p}}_{1}$ & -0.40 & -0.03 & 0.08 & 0.49 & 1 & 1 & -0.17 & -0.03 & 0.08 & 0.25 & 0.9 & 0.9 \\
\hline & $\widetilde{\widetilde{n p}}_{2}$ & -0.20 & 0.14 & 0.25 & 0.65 & 1 & 1 & 0.01 & 0.14 & 0.25 & 0.40 & 0.9 & 0.9 \\
\hline & $\widetilde{\widetilde{n p}}_{3}$ & -0.29 & 0.09 & 0.19 & 0.62 & 1 & 1 & -0.06 & 0.09 & 0.19 & 0.36 & 0.9 & 0.9 \\
\hline & $\widetilde{\widetilde{n g}}_{4}$ & -0.27 & 0.63 & 0.93 & 1.94 & 1 & 1 & 0.25 & 0.63 & 0.93 & 1.34 & 0.9 & 0.9 \\
\hline & $\widetilde{\widetilde{n p}}_{5}$ & -0.01 & 0.54 & 0.70 & 1.26 & 1 & 1 & 0.30 & 0.54 & 0.70 & 0.93 & 0.9 & 0.9 \\
\hline & $\widetilde{\widetilde{n p}}_{6}$ & 0.05 & 0.88 & 1.12 & 1.99 & 1 & 1 & 0.50 & 0.88 & 1.12 & 1.47 & 0.9 & 0.9 \\
\hline & $\widetilde{\widetilde{n p}}_{7}$ & -0.17 & 0.03 & 0.08 & 0.32 & 1 & 1 & -0.04 & 0.03 & 0.08 & 0.17 & 0.9 & 0.9 \\
\hline & $\widetilde{\widetilde{n p}}_{8}$ & -0.15 & 0.02 & 0.07 & 0.23 & 1 & 1 & -0.05 & 0.02 & 0.07 & 0.14 & 0.9 & 0.9 \\
\hline
\end{tabular}




\section{Ekonomika a management}

\begin{tabular}{|c|c|c|c|c|c|c|c|c|c|c|c|c|c|}
\hline \multirow{3}{*}{$\begin{array}{l}\text { Tab. } \\
\qquad \tilde{\tilde{K}}\end{array}$} & \multicolumn{13}{|c|}{$\begin{array}{l}\text { The weighted sum of distances, their normalized values and the appraisal } \\
\text { scores - Part } 2\end{array}$} \\
\hline & & \multicolumn{6}{|c|}{$\tilde{K}_{i j}^{U}$} & \multicolumn{6}{|c|}{$\tilde{K}_{i j}^{L}$} \\
\hline & & $k_{1}^{U}$ & $k_{2}^{U}$ & $k_{3}^{U}$ & $k_{4}^{U}$ & $H_{l}^{U}$ & $H_{2}^{U}$ & $k_{l}^{L}$ & $k_{2}^{L}$ & $k_{3}^{L}$ & $k_{4}^{L}$ & $H_{1}^{L}$ & $H_{2}^{L}$ \\
\hline \multirow{8}{*}{$11 ई$} & $\widetilde{\tilde{n n}}_{1}$ & -0.49 & 0.36 & 0.58 & 1.22 & 1 & 1 & 0.02 & 0.36 & 0.58 & 0.86 & 0.9 & 0.9 \\
\hline & $\widetilde{\widetilde{n n}}_{2}$ & -0.31 & 0.36 & 0.55 & 1.26 & 1 & 1 & 0.09 & 0.36 & 0.55 & 0.84 & 0.9 & 0.9 \\
\hline & $\widetilde{\widetilde{n n}}_{3}$ & -0.24 & 0.44 & 0.63 & 1.37 & 1 & 1 & 0.17 & 0.44 & 0.63 & 0.94 & 0.9 & 0.9 \\
\hline & $\widetilde{\widetilde{n n}}_{4}$ & 1.00 & 1.00 & 1.00 & 1.00 & 1 & 1 & 1.00 & 1.00 & 1.00 & 1.00 & 0.9 & 0.9 \\
\hline & $\widetilde{\widetilde{n n}}_{5}$ & 0.45 & 0.91 & 1.02 & 1.47 & 1 & 1 & 0.72 & 0.91 & 1.02 & 1.19 & 0.9 & 0.9 \\
\hline & $\widetilde{\tilde{n n}}_{6}$ & 1.00 & 1.00 & 1.00 & 1.00 & 1 & 1 & 1.00 & 1.00 & 1.00 & 1.00 & 0.9 & 0.9 \\
\hline & $\widetilde{\widetilde{n n}}_{7}$ & -0.50 & 0.46 & 0.72 & 1.63 & 1 & 1 & 0.06 & 0.46 & 0.72 & 1.08 & 0.9 & 0.9 \\
\hline & $\widetilde{\tilde{n n}}_{8}$ & -1.12 & -0.13 & 0.14 & 1.04 & 1 & 1 & -0.52 & -0.13 & 0.14 & 0.55 & 0.9 & 0.9 \\
\hline \multirow{8}{*}{ "16 } & $\tilde{\tilde{h}}_{1}$ & -0.44 & 0.17 & 0.33 & 0.85 & 1 & 1 & -0.08 & 0.17 & 0.33 & 0.55 & 0.9 & 0.9 \\
\hline & $\tilde{\tilde{h}}_{2}$ & -0.25 & 0.25 & 0.40 & 0.95 & 1 & 1 & 0.05 & 0.25 & 0.40 & 0.62 & 0.9 & 0.9 \\
\hline & $\tilde{\tilde{n}}_{3}$ & -0.27 & 0.27 & 0.41 & 0.99 & 1 & 1 & 0.06 & 0.27 & 0.41 & 0.65 & 0.9 & 0.9 \\
\hline & $\tilde{\tilde{h}}_{4}$ & 0.37 & 0.82 & 0.96 & 1.47 & 1 & 1 & 0.63 & 0.82 & 0.96 & 1.17 & 0.9 & 0.9 \\
\hline & $\tilde{\tilde{h}}_{5}$ & 0.22 & 0.72 & 0.86 & 1.36 & 1 & 1 & 0.51 & 0.72 & 0.86 & 1.06 & 0.9 & 0.9 \\
\hline & $\tilde{\tilde{h}}_{6}$ & 0.52 & 0.94 & 1.06 & 1.50 & 1 & 1 & 0.75 & 0.94 & 1.06 & 1.23 & 0.9 & 0.9 \\
\hline & $\tilde{\tilde{h}}_{7}$ & -0.33 & 0.25 & 0.40 & 0.97 & 1 & 1 & 0.01 & 0.25 & 0.40 & 0.62 & 0.9 & 0.9 \\
\hline & $\tilde{\tilde{h}}_{8}$ & -0.64 & -0.06 & 0.11 & 0.64 & 1 & 1 & -0.28 & -0.06 & 0.11 & 0.34 & 0.9 & 0.9 \\
\hline
\end{tabular}

\section{Tab. 10: The ranking values of appraisal scores}

\begin{tabular}{c|c} 
Alternatives & $(\mathcal{R V})$ \\
\hline $\mathcal{A}_{1}$ & 0.1633 \\
\hline $\mathcal{A}_{2}$ & 0.1767 \\
\hline $\mathcal{A}_{3}$ & 0.1778 \\
\hline $\mathcal{A}_{4}$ & 0.1957 \\
\hline $\mathcal{A}_{5}$ & 0.1938 \\
\hline $\mathcal{A}_{6}$ & 0.1964 \\
\hline $\mathcal{A}_{7}$ & 0.1753 \\
\hline $\mathcal{A}_{8}$ & 0.1049 \\
\hline
\end{tabular}


and Keshavarz Ghorabaee et al. (2015a). The symbols $\mathrm{M}-1$ to $\mathrm{M}-6$ are utilized for representing these selected methods, respectively.

For comparing the extended EDAS method with the other methods, the numerical example is solved using the selected methods separately, and the Spearman's rank correlation coefficient $\left(r_{s}\right)$ is used to analyze the ranking results. We can say that there is a significant statistical correlation between results if the values of $r$ are greater than 0.6 (Keshavarz Ghorabaee et al., 2016a). Tab. 11 represents the results of the comparison between the proposed method and the other methods. All correlation coefficients are greater than 0.6 according to this table. This fact demonstrates a strong or very strong relationship between the ranking results of the extended EDAS method and the other methods. Therefore, it can be said that the result of the proposed method is consistent with the results of other methods.

\begin{tabular}{|c|c|c|c|c|c|c|c|}
\hline \multirow{2}{*}{ Alternatives } & \multicolumn{7}{|c|}{ Methods } \\
\hline & $M-1$ & M-2 & M-3 & $M-4$ & M-5 & M-6 & EDAS-IT2FSs \\
\hline $\mathcal{A}_{1}$ & 7 & 7 & 7 & 7 & 7 & 4 & 7 \\
\hline $\mathcal{A}_{2}$ & 5 & 4 & 5 & 5 & 4 & 6 & 5 \\
\hline $\mathcal{A}_{3}$ & 4 & 5 & 6 & 4 & 5 & 7 & 4 \\
\hline $\mathcal{A}_{4}$ & 2 & 2 & 2 & 2 & 2 & 2 & 2 \\
\hline $\mathcal{A}_{5}$ & 3 & 3 & 3 & 3 & 3 & 3 & 3 \\
\hline $\mathcal{A}_{6}$ & 1 & 1 & 1 & 1 & 1 & 1 & 1 \\
\hline $\mathcal{A}_{7}$ & 6 & 6 & 4 & 6 & 6 & 5 & 6 \\
\hline $\mathcal{A}_{8}$ & 8 & 8 & 8 & 8 & 8 & 8 & 8 \\
\hline$r_{s}$ & 1 & 0.98 & 0.9 & 1 & 0.98 & 0.76 & - \\
\hline
\end{tabular}

Tab. 12: The generated weights for sensitivity analysis

\begin{tabular}{c|c|c|c|c|c|c|c}
\multirow{2}{*}{ Sets } & \multicolumn{7}{|c}{ Criteria } \\
\cline { 2 - 7 } & $\mathcal{C}_{1}$ & $\mathcal{C}_{2}$ & $\mathcal{C}_{3}$ & $\mathcal{C}_{4}$ & $\mathcal{C}_{5}$ & $\mathcal{C}_{6}$ & $\mathcal{C}_{7}$ \\
\hline 1 & 0.0357 & 0.0714 & 0.1071 & 0.1429 & 0.1786 & 0.2143 & 0.2500 \\
\hline 2 & 0.0714 & 0.1071 & 0.1429 & 0.1786 & 0.2143 & 0.2500 & 0.0357 \\
\hline 3 & 0.1071 & 0.1429 & 0.1786 & 0.2143 & 0.2500 & 0.0357 & 0.0714 \\
\hline 4 & 0.1429 & 0.1786 & 0.2143 & 0.2500 & 0.0357 & 0.0714 & 0.1071 \\
\hline 5 & 0.1786 & 0.2143 & 0.2500 & 0.0357 & 0.0714 & 0.1071 & 0.1429 \\
\hline 6 & 0.2143 & 0.2500 & 0.0357 & 0.0714 & 0.1071 & 0.1429 & 0.1786 \\
\hline 7 & 0.2500 & 0.0357 & 0.0714 & 0.1071 & 0.1429 & 0.1786 & 0.2143 \\
\hline
\end{tabular}


In addition to this comparison, a sensitivity analysis based on varying the weights of criteria is performed. For this aim, according to the number of criteria in this problem, we generate seven sets of criteria weights with a simple pattern. In the pattern which is used for sensitivity analysis, one criterion has the highest weight, and one criterion has the lowest weigh, and the other criteria have a weight between them. Using this pattern helps us to investigate the impact of changing the weights of criteria in a more effective way. The weights of criteria in each set are represented in Tab. 12.
As can be seen, a crisp weight is assigned to each criterion in these sets. The numerical example is solved with each set of generated weights separately. The ranking results with different sets are shown in Fig. 2. This figure represents less change in the rank of alternatives within different sets of generated criteria weights. Therefore, we can say that the proposed method has good stability when the weights of criteria are varied. These analyses demonstrate the validity and stability of the results of the extended EDAS method in the decision-making problem with multiple criteria and uncertain data.

\section{Fig. 2: The ranking results of the sensitivity analysis}

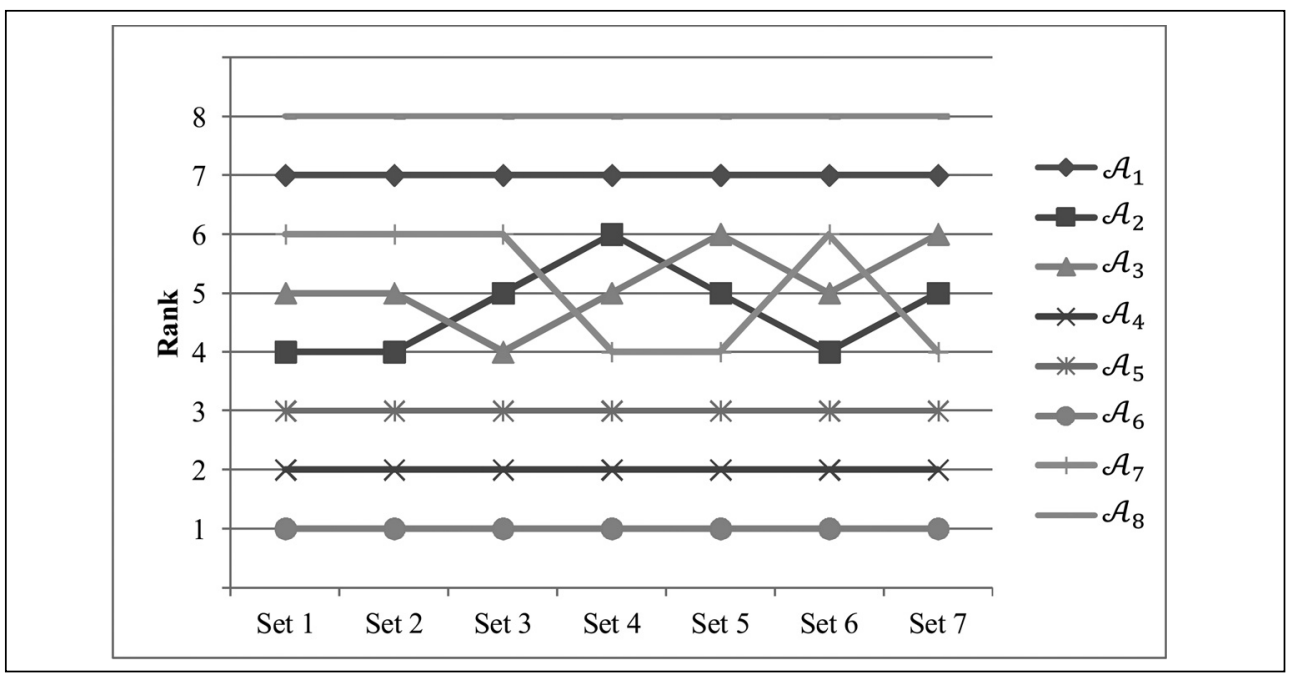

Source: own

\section{Conclusions}

The uncertainty is usually inevitable in the multi-criteria decision-making process. The fuzzy set theory is one of the efficient tools to deal with the MCDM problems in an uncertain environment. IT2FSs are flexible and give more degrees of freedom to decision-makers for modeling the decision-making problems. In this study, we have proposed an extended EDAS method with interval type-2 fuzzy sets. The concepts and arithmetic operations of IT2FSs have been utilized for extending this method. A numerical example of multi-criteria subcontractor evaluation problem has been used to illustrate the process of the proposed method. Moreover, to validate the results, this example has been solved by some existing methods. Also, we have performed a sensitivity analysis with seven sets of criteria weights. These sets have been designed according to the number of criteria in the problem, and a pattern has been used to generate the weights of criteria in each set. These analyses show that the results of the extended EDAS method are relatively consistent with the other methods of the comparison and have good stability 
in different sets of criteria weights. Future research can apply the proposed method to the MCDM problems such as supplier selection, robot selection, personnel selection, material selection and project selection.

\section{References}

Abbasianjahromi, H., Rajaie, H., \& Shakeri, E. (2013). A framework for subcontractor selection in the construction industry. Journal of Civil Engineering and Management, 19(2), 158168. doi:10.3846/13923730.2012.743922.

Aliakbari Nouri, F., Khalili Esbouei, S., \& Antucheviciene, J. (2015). A hybrid MCDM approach based on fuzzy ANP and fuzzy TOPSIS for technology selection. Informatica, 26(3), 369-388. doi:10.15388/ Informatica.2015.53.

Baležentis, T., \& Zeng, S. (2013). Group multi-criteria decision making based upon interval-valued fuzzy numbers: An extension of the MULTIMOORA method. Expert Systems with Applications, 40(2), 543-550. doi:10.1016/j. eswa.2012.07.066.

Balin, A., \& Baraçli, H. (2015). A fuzzy multicriteria decision making methodology based upon the interval type-2 fuzzy sets for evaluating renewable energy alternatives in Turkey. Technological and Economic Development of Economy. doi:10.3846/20294913.2015.1056276.

Celik, E., Gul, M., Aydin, N., Gumus, A. T., \& Guneri, A. F. (2015). A comprehensive review of multi criteria decision making approaches based on interval type-2 fuzzy sets. KnowledgeBased Systems, 85(September), 329-341. doi:10.1016/j.knosys.2015.06.004.

Celik, E., Gumus, A. T., \& Alegoz, M. (2014). A trapezoidal type-2 fuzzy MCDM method to identify and evaluate critical success factors for humanitarian relief logistics management. Journal of Intelligent \& Fuzzy Systems, 27(6), 2847-2855. doi:10.3233/IFS-141246.

Chakraborty, S., Zavadskas, E. K., \& Antucheviciene, J. (2015). Applications of waspas method as a multi-criteria decisionmaking tool. Economic Computation and Economic Cybernetics Studies and Research, 49(1), 1-17.

Chen, S.-M., \& Lee, L.-W. (2010). Fuzzy multiple attributes group decision-making based on the ranking values and the arithmetic operations of interval type-2 fuzzy sets. Expert Systems with Applications, 37(1), 824-833. doi:10.1016/j.eswa.2009.06.094.
Chen, S.-M., Yang, M.-W., Lee, L.-W., \& Yang, S.-W. (2012). Fuzzy multiple attributes group decision-making based on ranking interval type-2 fuzzy sets. Expert Systems with Applications, 39(5), 5295-5308. doi:10.1016/ j.eswa.2011.11.008.

Chen, T.-Y. (2015). Likelihoods of interval type-2 trapezoidal fuzzy preference relations and their application to multiple criteria decision analysis. Information Sciences, 295, 303-322. doi:10.1016/j.ins.2014.10.007.

Ecer, F. (2015). Performance evaluation of internet banking branches via a hybrid MCDM model under fuzzy environment. Economic Computation \& Economic Cybernetics Studies \& Research, 49(2), 200-218.

Faraji Sabokbar, H., Hosseini, A., Banaitis, A., \& Banaitiene, N. (2016). A novel sorting method TOPSIS-SORT: an applicaiton for Tehran environmental quality evaluation. E\&M Ekonomie a Management, 19(2), 87-104. doi:10.15240/tul/001/2016-2-006.

Hu, J., Zhang, Y., Chen, X., \& Liu, Y. (2013). Multi-criteria decision making method based on possibility degree of interval type-2 fuzzy number. Knowledge-Based Systems, 43(May), 21-29. doi:10.1016/j.knosys.2012.11.007.

Karabasevic, D., Zavadskas, E. K., Turskis, Z., \& Stanujkic, D. (2016). The framework for the selection of personnel based on the SWARA and ARAS methods under uncertainties. Informatica, 27(1), 49-65. doi:10.15388/ Informatica.2016.76.

Keshavarz Ghorabaee, M., Amiri, M., Salehi Sadaghiani, J., \& Hassani Goodarzi, G. (2014). Multiple criteria group decision-making for supplier selection based on COPRAS method with interval type-2 fuzzy sets. The International Journal of Advanced Manufacturing Technology, 75(5-8), 1115-1130. doi:10.1007/s00170-0146142-7.

Keshavarz Ghorabaee, M., Amiri, M., Sadaghiani, J. S., \& Zavadskas, E. K. (2015a). Multi-criteria project selection using an extended VIKOR method with interval type-2 fuzzy sets. International Journal of Information Technology \& Decision Making, 14(5), 9931016. doi:10.1142/S0219622015500212.

Keshavarz Ghorabaee, M., Zavadskas, E. K., Olfat, L., \& Turskis, Z. (2015b). Multi-criteria inventory classification using a new method of evaluation based on distance from average solution (EDAS). Informatica, 26(3), 435-451. doi:10.15388/Informatica.2015.57. 
Keshavarz Ghorabaee, M., Zavadskas, E. K., Amiri, M., \& Antucheviciene, J. (2016a). A new method of assessment based on fuzzy ranking and aggregated weights (AFRAW) for MCDM problems under type-2 fuzzy environment. Economic Computation and Economic Cybernetics Studies and Research, 50(1), 39-68.

Keshavarz Ghorabaee, M., Zavadskas, E. K., Amiri, M., \& Esmaeili, A. (2016b). Multicriteria evaluation of green suppliers using an extended WASPAS method with interval type-2 fuzzy sets. Journal of Cleaner Production, 137, 213-229. doi:10.1016/j.jclepro.2016.07.031.

Keshavarz Ghorabaee, M., Zavadskas, E. K., Amiri, M., \& Turskis, Z. (2016c). Extended EDAS Method for Fuzzy Multi-criteria Decision-making: An Application to Supplier Selection. International Journal of Computers Communications \& Control, 11(3), 358-371. doi:10.15837/ijccc.2016.3.2557.

Lazauskas, M., Zavadskas, E. K., \& Šaparauskas, J. (2015). Ranking of priorities among the baltic capital cities for the development of sustainable construction. E\&M Ekonomie a Management, 18(2), 15-24. doi:10.15240/tul/001/2015-2-002.

Li, G., Kou, G., \& Peng, Y. (2015). Dynamic fuzzy multiple criteria decision making for performance evaluation. Technological and Economic Development of Economy, 21(5), 705-719. doi:10.3846/20294913.2015.1056280.

Mahdiraji, H. A., Hajiagha, S. H. R., Hashemi, S. S., \& Zavadskas, E. K. (2016). A grey multi-objective linear model to find critical path of a project by using time, cost, quality and risk parameters. E\&M Ekonomie a Management, 19(1), 49-61. doi:10.15240/ tul/001/2016-1-004.

Mardani, A., Jusoh, A., \& Zavadskas, E. K. (2015). Fuzzy multiple criteria decision-making techniques and applications - Two decades review from 1994 to 2014. Expert Systems with Applications, 42(8), 4126-4148. doi:10.1016/j. eswa.2015.01.003.

Mardani, A., Jusoh, A., Zavadskas, E. K., Kazemilari, M., Ungku, N. U. A., \& Khalifah, Z. (2016). Application of Multiple Criteria Decision Making Techniques in Tourism and Hospitality Industry: a Systematic Review. Transformations in Business \& Economics, 15(1), 192-213.

Mendel, J. M., John, R. I., \& Feilong, L. (2006). Interval Type-2 Fuzzy Logic Systems Made Simple. Fuzzy Systems, IEEE
Transactions on Fuzzy Systems, 14(6), 808-821. doi:10.1109/TFUZZ.2006.879986.

Plebankiewicz, E. (2012). A fuzzy sets based contractor prequalification procedure. Automation in Construction, 22(March), 433-443. doi:10.1016/j.autcon.2011.11.003.

Razavi Hajiagha, S. H., Mahdiraji, H. A., Hashemi, S. S., \& Turskis, Z. (2015). Determining weights of fuzzy attributes for multi-attribute decision-making problems based on consensus of expert opinions. Technological and Economic Development of Economy, 21(5), 738-755. doi:10.3846/20294913.2015.1058301.

Sang, X., \& Liu, X. (2016). An interval type2 fuzzy sets-based TODIM method and its application to green supplier selection. Journal of the Operational Research Society, 67(5), 722-734. doi:10.1057/jors.2015.86.

Stanujkic, D., Zavadskas, E. K., \& Tamošaitienè, J. (2015). An approach to measuring website quality in the rural tourism industry based on atanassov intuitionistic fuzzy sets. E\&M Ekonomie a Management, 18(4), 184-199. doi:10.15240/tul/001/2015-4-013.

Tošenovský, F. (2015). Multicriteria decisionmaking weights and a competitive product design. E\&M Ekonomie a Management, 18(1), 84-94. doi:10.15240/tul/001/2015-1-007.

Triantaphyllou, E. (2013). Multi-criteria Decision Making Methods: A Comparative Study. New York: Springer. doi:10.1007/978-14757-3157-6.

Vahdani, B., Mousavi, S. M., Hashemi, H., Mousakhani, M., \& Tavakkoli-Moghaddam, R. (2013). A new compromise solution method for fuzzy group decision-making problems with an application to the contractor selection. Engineering Applications of Artificial Intelligence, 26(2), 779-788. doi:10.1016/j. engappai.2012.11.005.

Wang, W., Liu, X., \& Qin, Y. (2012). Multiattribute group decision making models under interval type-2 fuzzy environment. KnowledgeBased Systems, 30(June), 121-128. doi:10.1016/j.knosys.2012.01.005.

Yu, L., Yang, Z., \& Tang, L. (2016). PredictionBased Multi-Objective Optimization for Oil Purchasing and Distribution with the NSGA-II Algorithm. International Journal of Information Technology \& Decision Making, 15(2), 423-451. doi:10.1142/S0219622016500097.

Zadeh, L. A. (1965). Fuzzy sets. Information and Control, 8(3), 338-353. doi:10.1016/S00199958(65)90241-X. 
Zadeh, L. A. (1975). The concept of a linguistic variable and its application to approximate reasoning-I. Information Sciences, 8(3), 199-249. doi:10.1016/00200255(75)90036-5.

Zavadskas, E. K., Turskis, Z., \& Kildienè, S. (2014). State of art surveys of overviews on MCDM/MADM methods. Technological and Economic Development of Economy, 20(1), 165-179. doi:10.3846/20294913.2014.892037.

Mehdi Keshavarz Ghorabaee, Ph.D. Candidate Allameh Tabataba'i University Faculty of Management and Accounting Department of Industrial Management m.keshavarz_gh@yahoo.com
Prof. Maghsoud Amiri, Ph.D. Allameh Tabataba'i University Faculty of Management and Accounting Department of Industrial Management m.amiri.atu@gmail.com

Prof. Edmundas Kazimieras Zavadskas, Ph.D. Vilnius Gediminas Technical University Research Institute of Smart Building Technologies edmundas.zavadskas@vgtu.It

Prof. Zenonas Turskis, Ph.D. Vilnius Gediminas Technical University Research Institute of Smart Building Technologies zenonas.turskis@vgtu.It 


\title{
Abstract
}

\section{MULTI-CRITERIA GROUP DECISION-MAKING USING AN EXTENDED EDAS METHOD WITH INTERVAL TYPE-2 FUZZY SETS}

\author{
Mehdi Keshavarz Ghorabaee, Maghsoud Amiri, Edmundas Kazimieras \\ Zavadskas, Zenonas Turskis
}

Multi-criteria decision-making (MCDM) methods are very useful in the real-world decision-making problems. We are usually confronted with the decision-making process in an uncertain environment, and the fuzzy set theory is an efficient tool to handle this uncertainty. Interval type-2 fuzzy sets are one of the extensions of the fuzzy sets which are very flexible to model an uncertain environment. This study is related to MCDM problems within the context of interval type-2 fuzzy sets (IT2FSs). The evaluation based on distance from average solution (EDAS) method is a new and efficient MCDM method, and assessment of alternatives in this method is based on the distance of them from average solution with respect to all criteria. In the EDAS method, each alternative has positive and negative distances which are used to determine the appraisal score of it. In this research, we present an extended EDAS method, which is named EDAS-IT2FSs, for dealing with multicriteria group decision-making problems with interval type-2 fuzzy sets. Basic concepts of interval type-2 fuzzy sets and the arithmetic operations of trapezoidal IT2FSs are used to develop the extended EDAS method. A numerical example of multi-criteria subcontractor evaluation problem is used to illustrate the process of using the extended EDAS method. The example involves eight subcontractors that need to be evaluated with respect to seven criteria. A comparison and a sensitivity analysis based on different sets of criteria weights are also performed to show the validity of the proposed method. The results of these analyses show the efficiency and stability of the extended EDAS method.

Key Words: Multi-criteria decision-making, interval type-2 fuzzy sets, fuzzy MCDM, EDAS method.

JEL Classification: C02, C44, C61, C63, L7.

DOI: 10.15240/tul/001/2017-1-004 